ORIGINAL ARTICLE

\author{
P. Cannarsa - P. Cardaliaguet . \\ G. Crasta - E. Giorgieri
}

\title{
A boundary value problem for a PDE model in mass transfer theory: Representation of solutions and applications
}

Received: 10 July 2004 / Accepted: 16 December 2004 / Published online: 13 September 2005

(C) Springer-Verlag 2005

Abstract The system of partial differential equations

$$
\begin{cases}-\operatorname{div}(v D u)=f & \text { in } \Omega \\ |D u|-1=0 & \text { in }\{v>0\}\end{cases}
$$

arises in the analysis of mathematical models for sandpile growth and in the context of the Monge-Kantorovich optimal mass transport theory. A representation formula for the solutions of a related boundary value problem is here obtained, extending the previous two-dimensional result of the first two authors to arbitrary space dimension. An application to the minimization of integral functionals of the form

$$
\int_{\Omega}[h(|D u|)-f(x) u] \mathrm{d} x,
$$

with $f \geq 0$, and $h \geq 0$ possibly non-convex, is also included.

P. Cannarsa $(\varangle)$

Dipartimento di Matematica, Università di Roma Tor Vergata, Via della Ricerca Scientifica 1, 00133 Roma, Italy

E-mail: cannarsa@axp.mat.uniroma2.it

P. Cardaliaguet

Université de Bretagne Occidentale, UFR des Sciences et Techniques, 6 Av. Le Gorgeu, BP 809, 29285 Brest, France

E-mail: Pierre.Cardaliaguet@univ-brest.fr

G. Crasta

Dipartimento di Matematica, Università di Roma La Sapienza, P.le Aldo Moro 2,

00185 Roma, Italy

E-mail: crasta@mat.uniroma1.it

E. Giorgieri

Dipartimento di Matematica, Università di Roma Tor Vergata, Via della Ricerca Scientifica

1, 00133 Roma, Italy

E-mail: giorgier@axp.mat.uniroma2.it 
Keywords Granular matter · Eikonal equation - Singularities · Semiconcave functions · Viscosity solutions - Optimal mass transfer - Existence of minimizers . Distance function $\cdot$ Calculus of variations $\cdot$ Nonconvex integrands

Mathematics Subject Classification: Primary 35C15, 49J10, Secondary 35Q99, $49 \mathrm{~J} 30$

\section{Introduction}

This paper is concerned with the system of partial differential equations

$$
\begin{cases}-\operatorname{div}(v D u)=f & \text { in } \Omega \\ v \geq 0,|D u| \leq 1 & \text { in } \Omega \\ |D u|-1=0 & \text { in }\{v>0\}\end{cases}
$$

complemented with the conditions

$$
\begin{cases}u \geq 0, & \text { in } \Omega \\ u \equiv 0 & \text { on } \partial \Omega\end{cases}
$$

Here $\Omega \subset \mathbb{R}^{n}$ is a bounded domain with $\mathcal{C}^{2}$ boundary and $f \geq 0$ is a continuous function in $\Omega$.

The interest in the above problem can be motivated in several ways. For instance, (1)-(2) characterizes the equilibrium solutions of a system of PDEs introduced in [3] and in [17] as a dynamical model for granular matter. Moreover, system (1)-(2) plays the role of necessary conditions in the Monge-Kantorovich optimal mass transport theory, as shown in [12]. Problem (1)-(2) has been analyzed by the first two authors in [4] in the case of dimension $n=2$, obtaining a representation formula for the solution. In this paper we will generalize the result of [4] to an arbitrary space dimension, characterizing the unique solution (in a suitable weak sense) of (1)-(2).

To describe the result more precisely, let us introduce our notations. We denote by $d: \bar{\Omega} \rightarrow \mathbb{R}$ the distance function from the boundary of $\Omega$ and by $\Sigma$ the singular set of $d$, that is the set of points $x \in \Omega$ at which $d$ is not differentiable. Moreover, we indicate with $\Pi(\mathrm{x})$ the set of projections of $x$ onto $\partial \Omega$ and, when $x \in \Omega \backslash \Sigma$, we denote by $\kappa_{i}(\mathrm{x}), i \in\{1, \ldots, n-1\}$, the principal curvatures of $\partial \Omega$ at the (unique) projection $\Pi(\mathrm{x})$ of $x$ onto $\partial \Omega$. In our analysis, a major role will be played by the function

$$
\tau(x)=\min \{t \geq 0: x+t D d(x) \in \bar{\Sigma}\} \quad \forall x \in \bar{\Omega} \backslash \bar{\Sigma},
$$

which we call the maximal retraction length of $\Omega$ onto $\bar{\Sigma}$ or normal distance to $\bar{\Sigma}$.

We will prove that, in arbitrary space dimension, the unique solution of system (1)-(2) is given by the pair $\left(d, v_{f}\right)$, where $d$ is the distance function from the boundary $\partial \Omega$ and

$$
v_{f}(x)= \begin{cases}\int_{0}^{\tau(x)} f(x+t D d(x)) \prod_{i=1}^{n-1} \frac{1-(d(x)+t) \kappa_{i}(x)}{1-d(x) \kappa_{i}(x)} \mathrm{d} t & \forall x \in \Omega \backslash \bar{\Sigma}, \\ 0 & \forall x \in \bar{\Sigma} .\end{cases}
$$


The fact that relevant objects in Monge-Kantorovich theory can be represented in terms of the principal curvatures of $\partial \Omega$ is certainly not new in the literature. For instance, see the representation formula for the dynamic of a collapsing sandpile obtained first, in [11], by a formal computation, and then in [15] by a rigorous argument. Nevertheless, formula (3) is new-to our knowledge - and so is the existence of a continuous solution $v$ to (1)-(2), except for the case $n=2$ studied by the first two authors of this paper in [4].

Let us now compare the present work with its two-dimensional analogue [4]. On the one hand, showing that $\left(d, v_{f}\right)$ is a solution of (1)-(2) follows the same lines as in dimension two. On the other hand, the proof of the fact that $\left(d, v_{f}\right)$ is the unique solution to (1)-(2) requires completely different arguments. In fact, in dimension two one can exploit the relatively simple structure of $\bar{\Sigma}$ to show-by a direct argument-that any solution $(u, v)$ of (1)-(2) satisfies $u \equiv d$ in the set $\left\{x \in \Omega: v_{f}(x)>0\right\}$ and $v \equiv 0$ on $\bar{\Sigma}$. Such a technique cannot be extended to higher space dimension due to obvious topological obstructions.

In this paper, uniqueness is obtained as follows. To see that the first component of a solution of (1)-(2) is given by the distance function, we adapt an idea of [21], showing that $(u, v)$ is a saddle point of a suitable integral functional. Then, to identify the second component of $(u, v)$ with the function $v_{f}$, we compute the variation of $v$ along all rays $x+t D d(x), 0<t<\tau(x)$-which cover the set $\Omega \backslash \bar{\Sigma}$-as follows:

$$
\begin{aligned}
& v(x)-\prod_{i=1}^{n-1} \frac{1-(d(x)+t) \kappa_{i}(x)}{1-d(x) \kappa_{i}(x)} v(x+t D d(x)) \\
& =\int_{0}^{t} f(x+s D d(x)) \prod_{i=1}^{n-1} \frac{1-(d(x)+s) \kappa_{i}(x)}{1-d(x) \kappa_{i}(x)} \mathrm{d} s .
\end{aligned}
$$

Finally, using the fact that $v \equiv 0$ on $\bar{\Sigma}$-which can be proven by a blow up argument as in [12]—we easily deduce that $v \equiv v_{f}$ in $\Omega$.

We conclude this paper with an application to a problem in the Calculus of Variations which may seem quite unrelated to the present context at first glance.

Let us consider an integral functional of the form

$$
J_{0}(u)=\int_{\Omega}[h(|D u|)-f(x) u] \mathrm{d} x, \quad u \in W_{0}^{1,1}(\Omega),
$$

where $f \in L^{\infty}(\Omega)$ is a nonnegative function and $h:[0,+\infty) \rightarrow[0,+\infty]$ is a lower semicontinuous function (possibly with non-convex values) satisfying

$$
h(R)=0, \quad h(s) \geq \max \{0, \Lambda(s-R)\} \quad \text { for some constants } R, \Lambda>0 .
$$

In a pioneering work [8], A. Cellina proved that, if $\Omega$ is a convex domain (that is, an open bounded convex set) in $\mathbb{R}^{2}$ with piecewise smooth $\left(C^{2}\right)$ boundary and $f \equiv 1$, then $J_{0}$ does attain its minimum in $W_{0}^{1,1}(\Omega)$, and a minimizer is explicitly given by the function

$$
u_{\Omega}(x)=R d(x), \quad x \in \Omega,
$$

provided that the inradius $r_{\Omega}$ of $\Omega$ is small enough. (We recall that $r_{\Omega}$ is the supremum of the radii of all balls contained in $\Omega$.) This result has been extended to 
convex domains in $\mathbb{R}^{n}$ and to more general functionals in subsequent works (see $[6,7,10,23,24])$. One common point of all these results is that the set $\Omega$ is always a convex subset of $\mathbb{R}^{n}$.

In this paper we will prove that the function $u_{\Omega}$ defined in (7) is a minimizer of the functional $J_{0}$ in $W_{0}^{1,1}(\Omega)$ even on possibly nonconvex domains $\Omega$. This extension to nonconvex domains will be obtained as a consequence of the representation formula for the solution of system (1)-(2).

This paper is organized as follows. Section 2 is concerned with notations and preliminary results. In Sect. 3 we show that $\left(d, v_{f}\right)$ is a solution of system (1)-(2) in a suitable weak sense. Section 4 is devoted to the proof of uniqueness of such a pair as a solution of system (1)-(2). Finally, Sect. 5 contains our application to the existence of distance-like minimizers for the class of integral functionals (5).

\section{Preliminaries and Notations}

Most of the results of this section and of the following one are a simple generalization of those given in [4]. So, whenever we omit a proof, we understand a reference to [4].

Let $\Omega$ be a bounded domain with $\mathcal{C}^{2}$ boundary $\partial \Omega$. In what follows we denote by $d: \bar{\Omega} \rightarrow \mathbb{R}$ the distance function from the boundary of $\Omega$ and by $\Sigma$ the singular set of $d$, that is, the set of points $x \in \Omega$ at which $d$ is not differentiable. Since $d$ is Lipschitz continuous, $\Sigma$ has Lebesgue measure zero. Introducing the projection $\Pi(x)$ of $x$ onto $\partial \Omega$ in the usual way, $\Sigma$ is also the set of points $x$ at which $\Pi(x)$ is not a singleton.

For any $x \in \partial \Omega$ and $i=1, \ldots, n-1$, the number $\kappa_{i}(x)$ denotes the $i$ th principal curvature of $\partial \Omega$ at the point $x$, corresponding to a principal direction $e_{i}(x)$ orthogonal to $\operatorname{Dd}(x)$, with the sign convention $\kappa_{i} \geq 0$ if the normal section of $\Omega$ along the direction $e_{i}$ is convex. Also, we will label in the same way the extension of $\kappa_{i}$ to $\bar{\Omega} \backslash \Sigma$ given by

$$
\kappa_{i}(x)=\kappa_{i}(\Pi(x)) \quad \forall x \in \bar{\Omega} \backslash \Sigma .
$$

In the result below, $p \otimes q$ stands for the tensor product of two vectors $p, q \in$ $\mathbb{R}^{n}$, defined as $(p \otimes q)(x)=p\langle q, x\rangle, \forall x \in \mathbb{R}^{n}$. We refer to [16] for the proof of the representation formula for the Hessian matrix of $\mathrm{d}$.

Proposition 2.1 For any $x \in \bar{\Omega}$ and any $y \in \Pi(x)$ we have

$$
\kappa_{i}(y) d(x) \leq 1 \quad \forall i=1, \ldots, n-1 .
$$

If, in addition, $x \in \bar{\Omega} \backslash \bar{\Sigma}$, then

$$
\kappa_{i}(x) d(x)<1 \quad \text { and } \quad D^{2} d(x)=-\sum_{i=1}^{n-1} \frac{\kappa_{i}(x)}{1-\kappa_{i}(x) d(x)} e_{i}(x) \otimes e_{i}(x)
$$

where $e_{i}(x)$ is the unit eigenvector corresponding to $\frac{\kappa_{i}(x)}{1-\kappa_{i}(x) d(x)}$. 
Remark 2.2 Note that the regularity of $\Omega$ guarantees that the principal curvatures $\kappa_{i}$ are continuous functions on $\partial \Omega$.

The set $\Gamma$ of points $x \in \Omega \backslash \Sigma$ such that the equality sign holds in (2.1) for some index $i$ is called the set of regular conjugate points. It represents, in the sense explained in the proposition below, the "boundary" of the singular set $\Sigma$.

Proposition 2.2 Under the assumption that $\Omega$ is a bounded domain with $\mathcal{C}^{2}$ boundary $\partial \Omega$, we have $\bar{\Sigma} \subset \Omega$ and $\bar{\Sigma}=\Sigma \cup \Gamma$.

Now, let us focus on the function

$$
\tau(x)=\left\{\begin{array}{lc}
\min \{t \geq 0: x+t \operatorname{Dd}(x) \in \bar{\Sigma}\} & \forall x \in \bar{\Omega} \backslash \bar{\Sigma}, \\
0 & \forall x \in \bar{\Sigma} .
\end{array}\right.
$$

Since the map $x \mapsto x+\tau(x) D d(x)$ is a natural retraction of $\bar{\Omega}$ onto $\bar{\Sigma}$, we will refer to $\tau(\cdot)$ as the maximal retraction length of $\Omega$ onto $\bar{\Sigma}$ or normal distance to $\bar{\Sigma}$.

Let us analyze the regularity properties of $\tau$. The first theorem is a fine regularity result due to Li and Nirenberg (see [19]).

Theorem 2.4 Let $\Omega$ be a bounded domain in $\mathbb{R}^{n}$ with boundary of class $\mathcal{C}^{2,1}$. Then the map $\tau$ defined in (9) is Lipschitz continuous on $\partial \Omega$.

Hereafter, we will denote by $\operatorname{Lip}(\tau)$ the Lipschitz semi-norm of $\tau$ on $\partial \Omega$. Since $x \mapsto x+\tau(x) D d(x)$ maps $\partial \Omega$ onto $\bar{\Sigma}$, a straightforward application of Theorem 2.4 is that the $(n-1)$-dimensional Hausdorff measure of $\bar{\Sigma}$ is finite:

Corollary 2.5 Let $\Omega$ be a bounded domain in $\mathbb{R}^{n}$ with boundary of class $\mathcal{C}^{2,1}$. Then,

$$
\mathcal{H}^{n-1}(\bar{\Sigma}) \leq k_{\Omega} \mathcal{H}^{n-1}(\partial \Omega)<\infty
$$

where $k_{\Omega} \geq 0$ is a constant depending on $\operatorname{Lip}(\tau)$ and $\Omega$.

For less regular domains the Lipschitz continuity of $\tau$ may fail, but continuity is preserved.

Lemma 2.6 Assume that $\Omega$ is a connected bounded open subset of $\mathbb{R}^{n}$ with $\mathcal{C}^{2}$ boundary. Then the map $\tau$, extended to 0 on $\bar{\Sigma}$, is continuous in $\bar{\Omega}$.

We now give an approximation result that guarantees the stability of the singular set and of the maximal retraction length with respect to the convergence in the $\mathcal{C}^{2}$ topology. Let us first define the signed distance from $\partial \Omega$ as

$$
b_{\Omega}(x)= \begin{cases}d_{\partial \Omega}(x) & \text { if } \quad x \in \mathbb{R}^{n} \backslash \bar{\Omega} \\ -d_{\partial \Omega}(x) & \text { if } \quad x \in \bar{\Omega}\end{cases}
$$

We say that a sequence of bounded domains $\left\{\Omega_{k}\right\}$ with $\mathcal{C}^{2}$ boundary converges to $\Omega$ in the $\mathcal{C}^{2}$ topology if $b_{\Omega_{k}}, D b_{\Omega_{k}}$ and $D^{2} b_{\Omega_{k}}$ converge to $b_{\Omega}, D b_{\Omega}$ and $D^{2} b_{\Omega}$, uniformly in a neighborhood of $\partial \Omega$. 
Proposition 2.7 Let $\left\{\Omega_{k}\right\}$ be a sequence of bounded domains with $\mathcal{C}^{2}$ boundary. For any $k \in \mathbb{N}$, denote by $\Sigma_{k}$ and $\tau_{k}$, respectively, the singular set and maximal retraction length of $\Omega_{k}$. If $\left\{\Omega_{k}\right\}$ converges to $\Omega$ in the $\mathcal{C}^{2}$ topology, then $\left\{\bar{\Sigma}_{k}\right\}$ converges to $\bar{\Sigma}$ in the Hausdorff topology, and $\left\{\tau_{k}\right\}$ converges to $\tau$ uniformly on all compact subsets of $\Omega$.

We conclude this section with the definition of viscosity solutions for the eikonal equation and the connection with the distance function $d$ from $\Omega$.

For any measurable set $A \subset \mathbb{R}^{n}$, we denote by $|A|$ the Lebesgue measure of $A$. If $u: A \rightarrow \mathbb{R}$ is a bounded measurable function, then $\|u\|_{\infty, A}$ stands for the essential supremum of $u$ in $A$. If $A$ is open and $u$ is Lipschitz continuous, then, by Rademacher's Theorem, $u$ is differentiable a. e. in $A$. In this case, we denote by $\|D u\|_{\infty, A}$ the number $\sup \{|D u(x)|: x \in A, \exists D u(x)\}$, and by $D^{*} u(x)$ the set of limiting gradients of $u$ at $x$ defined as

$$
D^{*} u(x)=\left\{\lim _{k} D u\left(x_{k}\right): A \ni x_{k} \rightarrow x, \quad \exists D u\left(x_{k}\right)\right\} .
$$

As usual, the superdifferential of $u$ at a point $x \in A$ is the set

$$
D^{+} u(x)=\left\{p \in \mathbb{R}^{n} \mid \limsup _{h \rightarrow 0} \frac{u(x+h)-u(x)-\langle p, h\rangle}{|h|} \leq 0\right\},
$$

while the subdifferential $D^{-} u$ is given by the formula $D^{-} u(x)=-D^{+}(-u)(x)$.

Definition 2.8 We say that $u$ is a viscosity solution of the eikonal equation $|D u|=$ 1 in an open set $\Omega \subset \mathbb{R}^{n}$ if, for any $x \in \Omega \subset \mathbb{R}^{n}$, we have

$$
\begin{aligned}
& p \in D^{-} u(x):|p| \geq 1, \\
& p \in D^{+} u(x):|p| \leq 1 .
\end{aligned}
$$

We recall that the distance function $d$ is the unique viscosity solution of the eikonal equation $|D u|=1$ in $\Omega$, with boundary condition $u=0$ in $\partial \Omega$. Equivalently, $d$ is the largest function such that $\|D u\|_{\infty, \Omega} \leq 1$ and $u=0$ on $\partial \Omega$. The reader is referred to [2] and to [20] for a detailed discussion on these topics.

\section{Existence}

In this section we prove that the pair $\left(d, v_{f}\right)$, where $d$ is the distance function from $\partial \Omega$ and

$$
v_{f}(x)=\left\{\begin{array}{lc}
\int_{0}^{\tau(x)} f(x+t D d(x)) \prod_{i=1}^{n-1} \frac{1-(d(x)+t) \kappa_{i}(x)}{1-d(x) \kappa_{i}(x)} \mathrm{d} t & \forall x \in \Omega \backslash \bar{\Sigma}, \\
0 & \forall x \in \bar{\Sigma},
\end{array}\right.
$$

is a solution of system (1)-(2). More precisely, we will prove the following result.

Theorem 3.1 Let $\Omega \subset \mathbb{R}^{n}$ be a bounded domain with boundary of class $\mathcal{C}^{2}$ and $f \geq 0$ be a bounded continuous function in $\Omega$. Then, the pair $\left(d, v_{f}\right)$ defined above satisfies (1)-(2) in the following sense: 
1. $\left(d, v_{f}\right)$ is a pair of bounded continuous functions

2. $d=0$ on $\partial \Omega,\|D d\|_{\infty, \Omega} \leq 1$, and $d$ is a viscosity solution of

$$
|D u|=1 \quad \text { in } \quad\left\{x \in \Omega: v_{f}(x)>0\right\}
$$

3. $v_{f} \geq 0$ in $\Omega$ and, for every test function $\phi \in \mathcal{C}_{c}^{\infty}(\Omega)$,

$$
\int_{\Omega} v_{f}(x)\langle D d(x), D \phi(x)\rangle \mathrm{d} x=\int_{\Omega} f(x) \phi(x) \mathrm{d} x .
$$

We begin with two preliminary results, the former describing continuity and differentiability properties of $v_{f}$, the latter providing an approximation result for the characteristic function of a compact set, in the spirit of capacity theory.

Proposition 3.2 Let $\Omega \subset \mathbb{R}^{n}$ be a bounded domain with boundary of class $\mathcal{C}^{2}$ and $f \geq 0$ be a continuous function in $\Omega$. Then, $v_{f}$ is a locally bounded continuous function in $\Omega$. Moreover, in any set $\Omega_{\varepsilon}:=\{x \in \Omega: d(x)>\varepsilon\}, \varepsilon>0$, $v_{f}$ satisfies the bound

$$
0 \leq v_{f}(x) \leq\|f\|_{\infty, \Omega_{\varepsilon}} \prod_{i=1}^{n-1}\left[1+\left\|\left[\kappa_{i}\right]_{-}\right\|_{\mathcal{C}(\partial \Omega)} \operatorname{diam} \Omega\right] \tau(x) \quad \forall x \in \Omega_{\varepsilon},
$$

where $\left\|\left[\kappa_{i}\right]_{-}\right\|_{\mathcal{C}(\partial \Omega)}:=\max _{x \in \partial \Omega}\left[\kappa_{i}(x)\right]_{-}$. If, in addition, $\partial \Omega$ is of class $\mathcal{C}^{2,1}$ and $f$ is Lipschitz continuous in $\Omega$, then $v_{f}$ is locally Lipschitz continuous in $\Omega \backslash \bar{\Sigma}$ and satisfies

$$
-\operatorname{div}\left(v_{f}(x) D d(x)\right)=f(x)
$$

at each point $x \in \Omega \backslash \bar{\Sigma}$ at which $v_{f}$ is differentiable.

Remark 3.3 Since $d$ is $\mathcal{C}^{2}$ in $\Omega \backslash \bar{\Sigma}$, equality (13) reads

$$
\left\langle D v_{f}(x), D d(x)\right\rangle+v_{f}(x) \Delta d(x)+f(x)=0 .
$$

Moreover, a straightforward consequence of Proposition 3.2 is that the equality $-\operatorname{div}\left(v_{f} D d\right)=f$ holds in the sense of distributions in $\Omega \backslash \bar{\Sigma}$ as soon as $f$ is Lipschitz and $\partial \Omega$ of class $\mathcal{C}^{2,1}$.

Remark 3.4 A sharper upper bound on $v_{f}$ will be proven in Proposition 5.9.

Proof We note, first, that the maps $D d, \tau$ and $\kappa_{i}$ are continuous in $\Omega \backslash \bar{\Sigma}$ since $\Omega$ has a $\mathcal{C}^{2}$ boundary. Hence, when $f$ is continuous, so is $v_{f}$ in $\Omega \backslash \bar{\Sigma}$.

Let us now prove that $v_{f}$ is continuous on $\bar{\Sigma}$. Observe that, for any $x \notin \bar{\Sigma}$, the term

$$
\frac{1-(d(x)+t) \kappa_{i}(x)}{1-d(x) \kappa_{i}(x)}=\frac{1-d(x+t D d(x)) \kappa_{i}(x)}{1-d(x) \kappa_{i}(x)} \quad 0<t<\tau(x)
$$

is nonnegative by Proposition 2.1. A simple computation shows that it is also bounded by $\prod_{i=1}^{n-1}\left[1+\left\|\left[\kappa_{i}\right]_{-}\right\|_{\mathcal{C}(\partial \Omega)}\right] \tau(x)$. This proves (12) recalling that $x+t D d(x) \in \Omega_{\varepsilon}$ whenever $x \in \Omega_{\varepsilon}$ and $0 \leq t \leq \tau(x)$. The continuity of $v_{f}$ on $\bar{\Sigma}$ is an immediate consequence of (12). 
Next, let $\partial \Omega$ be of class $\mathcal{C}^{2,1}$ and $f$ be Lipschitz continuous. Then, Theorem 2.4 ensures that $\tau$ is Lipschitz continuous on $\partial \Omega$. Therefore, $\tau=\tau \circ \Pi$ is locally Lipschitz continuous in $\bar{\Omega} \backslash \bar{\Sigma}$, as well as $v_{f}$.

Finally, let us check the validity of (13) at every differentiability point $x$ for $v_{f}$ in the open set $\Omega \backslash \bar{\Sigma}$. Set $e_{n}:=\operatorname{Dd}(x)$ and consider $\left\{e_{1}, \ldots, e_{n}\right\}$ as a coordinate system, where $e_{i}=e_{i}(x), i=1, \ldots, n-1$ are the unit eigenvectors corresponding to the principal curvatures of $\partial \Omega$ at the projection point of $x$ on the boundary. We note that, at any such point $x$,

$$
\left\langle D v_{f}(x), D d(x)\right\rangle=\left.\frac{d}{d \lambda} v_{f}(x+\lambda D d(x))\right|_{\lambda=0} .
$$

But $\tau(x+\lambda D d(x))=\tau(x)-\lambda$ and $d(x+\lambda D d(x))=d(x)+\lambda$ for $\lambda>0$ sufficiently small. So,

$$
\begin{aligned}
& v_{f}(x+\lambda D d(x)) \\
& =\int_{0}^{\tau(x)-\lambda} f(x+(t+\lambda) D d(x)) \prod_{i=1}^{n-1} \frac{1-(d(x)+\lambda+t) \kappa_{i}(x)}{1-(d(x)+\lambda) \kappa_{i}(x)} \mathrm{d} t \\
& =\int_{\lambda}^{\tau(x)} f(x+t D d(x)) \prod_{i=1}^{n-1} \frac{1-(d(x)+t) \kappa_{i}(x)}{1-(d(x)+\lambda) \kappa_{i}(x)} \mathrm{d} t .
\end{aligned}
$$

Therefore,

$$
\begin{aligned}
& \left\langle D v_{f}(x), D d(x)\right\rangle \\
& =\int_{0}^{\tau(x)} f(x+t D d(x)) \sum_{i=1}^{n-1}\left[\frac{1-(d(x)+t) \kappa_{i}(x)^{2}}{\left(1-d(x) \kappa_{i}(x)\right)^{2}}\right. \\
& \left.\cdot \prod_{\substack{j=1 \\
j \neq i}}^{n-1} \frac{1-(d(x)+t) \kappa_{j}(x)}{1-d(x) \kappa_{j}(x)}\right] \mathrm{d} t-f(x) \\
& =\int_{0}^{\tau(x)} f(x+t D d(x))\left[\sum_{i=1}^{n-1} \frac{\kappa_{i}(x)}{\left(1-d(x) \kappa_{i}(x)\right)}\right] \prod_{i=1}^{n-1} \frac{1-(d(x)+t) \kappa_{i}(x)}{1-d(x) \kappa_{i}(x)} \mathrm{d} t \\
& -f(x) \\
& =-v_{f}(x) \Delta d(x)-f(x)
\end{aligned}
$$

where we have taken into account the identity

$$
\Delta d(x)=-\sum_{i=1}^{n-1} \frac{\kappa_{i}(x)}{\left(1-d(x) \kappa_{i}(x)\right)} \quad \forall x \in \Omega \backslash \bar{\Sigma},
$$

that follows from Proposition 2.1. We have thus obtained (14)—an equivalent version of (13) — and completed the proof. 
Proposition 3.3 Let $K$ be a compact subset of $\mathbb{R}^{n}$ such that $\mathcal{H}^{n-1}(K)<\infty$. Then, there exists a sequence $\left\{\xi_{k}\right\}$ of functions in $W^{1,1}\left(\mathbb{R}^{n}\right)$ with compact support, such that

(a) $0 \leq \xi_{k} \leq 1$ for every $k \in \mathbb{N}$;

(b) $d\left(\mathbb{R}^{n} \backslash \operatorname{spt}\left(\xi_{k}\right), K\right) \rightarrow 0$ as $k \rightarrow \infty$;

(c) $K \subset \operatorname{int}\left\{x \in \mathbb{R}^{n}: \xi_{k}(x) \geq 1\right\}$ for every $k \in \mathbb{N}$;

(d) $\xi_{k} \rightarrow 0$ in $L^{1}\left(\mathbb{R}^{n}\right)$ as $k \rightarrow \infty$;

(e) $\int_{\mathbb{R}^{n}}\left|D \xi_{k}\right| d x \leq C$ for every $k \in \mathbb{N}$ and some constant $C>0$.

The standard notations $d$, spt and int stand for distance (between two sets), support (of a function) and interior (of a set), respectively. We give a proof of the proposition for the reader's convenience.

Proof Since $\mathcal{H}^{n-1}(K)<\infty$, for any fixed $k \in \mathbb{N}$ there exists a sequence of points $\left\{x_{i}^{(k)}\right\}_{i \in \mathbb{N}}$ in $K$ and a sequence of radii $\left\{r_{i}^{(k)}\right\}_{i \in \mathbb{N}}$ such that

$-0<r_{i}^{(k)} \leq \frac{1}{k}$ and $\sum_{i}\left(r_{i}^{(k)}\right)^{n-1} \leq C\left(\mathcal{H}^{n-1}(K)+\frac{1}{k}\right) ;$

- $K \subset \operatorname{int}\left(\bigcup_{i} B_{r_{i}^{(k)}}\left(x_{i}^{(k)}\right)\right)$

for some constant $C>0$. Now, define, for any $x \in \mathbb{R}^{n}$,

$$
\begin{aligned}
\xi_{i}^{(k)}(x) & =\left[1-\frac{1}{r_{i}^{(k)}}\left(\left|x-x_{i}^{(k)}\right|-r_{i}^{(k)}\right)_{+}\right]_{+} \\
\xi_{k}(x) & =\sup _{i \in \mathbb{N}} \xi_{i}^{(k)}(x)
\end{aligned}
$$

and observe that

$$
\begin{aligned}
\operatorname{spt}\left(\xi_{i}^{(k)}\right) & =\bar{B}_{2 r_{i}^{(k)}}\left(x_{i}^{(k)}\right) \\
\operatorname{spt}\left(D \xi_{i}^{(k)}\right) & =\bar{B}_{2 r_{i}^{(k)}}\left(x_{i}^{(k)}\right) \backslash B_{r_{i}^{(k)}}\left(x_{i}^{(k)}\right) .
\end{aligned}
$$

Then, $\xi_{k} \in L^{1}\left(\mathbb{R}^{n}\right)$ since $0 \leq \xi_{k} \leq 1$ and $\xi_{k}$ has compact support. Moreover, an easy computation shows that $\int_{\mathbb{R}^{n}}\left|D \xi_{i}^{(k)}\right| d x=\omega_{n}\left(2^{n}-1\right)\left(r_{i}^{(k)}\right)^{n-1}$, where $\omega_{n}$ is the Lebesgue measure of the unit ball in $\mathbb{R}^{n}$. So, applying [13] Lemma 2, p. 148, we have

$$
\int_{\mathbb{R}^{n}}\left|D \xi_{k}\right| d x \leq \sup _{i} \int_{\mathbb{R}^{n}}\left|D \xi_{i}^{(k)}\right| d x \leq \sum_{i} \int_{\mathbb{R}^{n}}\left|D \xi_{k}^{(k)}\right| d x \leq C\left(\mathcal{H}^{n-1}(K)+\frac{1}{k}\right)
$$

for some constant $C>0$. Therefore, $\xi_{k} \in W^{1,1}\left(\mathbb{R}^{n}\right)$ and (e) holds true. Properties (b) and (c) are true by construction. Finally, (d) follows by Lebesgue Theorem because $0 \leq \xi_{k} \leq 1$ and $\xi_{k}(x)=0$ for any point $x \notin K$ and $k$ large enough.

Proof of Theorem 3.1 Let us first suppose that $\partial \Omega$ is of class $\mathcal{C}^{2,1}$ and $f$ is Lipschitz continuous in $\Omega$. We will prove that the pair $\left(d, v_{f}\right)$, with $v_{f}$ defined by (10), is a solution of system (1)-(2). Let us point out, to begin with, that $d$ is a viscosity 
solution of the eikonal equation in $\Omega$, and so, a fortiori, in the open set $\{x \in \Omega$ : $\left.v_{f}(x)>0\right\}$. Therefore, what actually remains to be shown is that

$$
\int_{\Omega} f \phi \mathrm{d} x=\int_{\Omega} v_{f}\langle D d, D \phi\rangle \mathrm{d} x \quad \forall \phi \in \mathcal{C}_{c}^{\infty}(\Omega) .
$$

Since $\mathcal{H}^{n-1}(\bar{\Sigma})<\infty$ by Proposition 2.5 , we can apply Proposition 3.3 with $K=\bar{\Sigma}$ to construct a sequence $\left\{\xi_{k}\right\}$ enjoying properties (a), (b), (c) and (d). Let $\phi \in \mathcal{C}_{c}^{\infty}(\Omega)$ be a test function, and set $\phi_{k}=\phi\left(1-\xi_{k}\right)$. Notice that, for $k$ large enough, $\operatorname{spt}\left(\phi_{k}\right) \subset \subset \Omega \backslash \bar{\Sigma}$. This follows from (a), (b) and from the fact that $\bar{\Sigma} \subset \Omega$ (see Proposition 2.2). Then, Proposition 3.2 and Rademacher's Theorem imply that $-\operatorname{div}\left(v_{f} D d\right)=f$ a. e. in $\Omega \backslash \bar{\Sigma}$. So, multiplying this equation by $\phi_{k}$ and integrating by parts, we obtain

$$
\int_{\Omega} f \phi_{k} \mathrm{~d} x=\int_{\Omega} v_{f}\left(1-\xi_{k}\right)\langle D d, D \phi\rangle \mathrm{d} x-\int_{\Omega} v_{f} \phi\left\langle D d, D \xi_{k}\right\rangle \mathrm{d} x .
$$

We claim that the rightmost term above goes to 0 as $k \rightarrow \infty$. Indeed,

$$
\begin{aligned}
\left|\int_{\Omega} v_{f} \phi\left\langle D d, D \xi_{k}\right\rangle \mathrm{d} x\right| & \leq\|\phi\|_{\infty, \Omega}\left\|v_{f}\right\|_{\infty, \operatorname{spt}\left(\xi_{k}\right)} \int_{\Omega}\left|D \xi_{k}\right| \mathrm{d} x \\
& \leq C\|\phi\|_{\infty, \Omega}\left\|v_{f}\right\|_{\infty, \operatorname{spt}\left(\xi_{k}\right)}
\end{aligned}
$$

where $C$ is the constant provided by Proposition 3.3 (d). Now, using property (a) of the proposition and the fact that $v_{f}$ is a continuous function vanishing on $\bar{\Sigma}$, we conclude that $\left\|v_{f}\right\|_{\infty, \operatorname{spt}\left(\xi_{k}\right)} \rightarrow 0$ as $k \rightarrow \infty$. This proves our claim. The conclusion (16) immediately follows since, in view of (a) and (c), the integrals $\int_{\Omega} f \phi_{k} \mathrm{~d} x$ and $\int_{\Omega} v_{f}\left(1-\xi_{k}\right)\langle D d, D \phi\rangle \mathrm{d} x$ converge to $\int_{\Omega} f \phi \mathrm{d} x$ and $\int_{\Omega} v_{f}\langle D d, D \phi\rangle \mathrm{d} x-$ respectively-as $k \rightarrow \infty$.

Finally, the extra assumptions that $\partial \Omega$ be of class $\mathcal{C}^{2,1}$ and $f$ be Lipschitz in $\Omega$, can be easily removed by an approximation argument based on the lemma below. Let $\left\{\Omega_{k}\right\}$ be a sequence of open domains, with $\mathcal{C}^{2,1}$ boundary, converging to $\Omega$ in the $\mathcal{C}^{2}$ topology, and let $\left\{f_{k}\right\}$ be a sequence of Lipschitz functions in $\Omega_{k}$ converging to $f$, uniformly on all compact subsets of $\Omega$. Denote by $\Sigma_{k}$ and $\tau_{k}$, respectively, the singular set and maximal retraction length of $\Omega_{k}$. Define $v_{k}(x)=0$ for every $x \in \bar{\Sigma}_{k}$ and

$$
v_{k}(x)=\int_{0}^{\tau_{k}(x)} f_{k}\left(x+t D d_{k}(x)\right) \prod_{i=1}^{n-1} \frac{1-\left(d_{k}(x)+t\right) \kappa_{k, i}(x)}{1-d_{k}(x) \kappa_{k, i}(x)} \mathrm{d} t \quad \forall x \in \Omega_{k} \backslash \bar{\Sigma}_{k},
$$

where $\kappa_{k, i}(x)$ stands for the $i$ th principal curvature of $\partial \Omega_{k}$ at the projection of $x$.

Lemma 3.6 $\left\{v_{k}\right\}$ converges to $v_{f}$ in $L_{l o c}^{1}(\Omega)$.

Proof Since, owing to (12), the sequence $\left\{v_{k}\right\}$ is locally uniformly bounded in $\Omega$, it suffices to prove that it converges uniformly to $v_{f}$ on every compact subset of $\Omega$. For this, recall that, on account of Proposition $2.7,\left\{\bar{\Sigma}_{k}\right\}$ converges to $\bar{\Sigma}$ in the Hausdorff topology and $\left\{\tau_{k}\right\}$ converges to $\tau$ uniformly on all compact subsets of $\Omega$. Then, our assumptions imply that $\left\{\kappa_{k, i}\right\}$ converges to $\kappa_{i}$ uniformly on every 
compact subset of $\Omega \backslash \bar{\Sigma}$ for any $i \in\{1, \ldots, n-1\}$, and so does $\left\{v_{k}\right\}$ to $v_{f}$. To complete the proof it suffices to combine the above local uniform convergence in $\Omega \backslash \bar{\Sigma}$ with the estimate

$$
0 \leq v_{k}(x) \leq\left\|f_{k}\right\|_{\infty, \Omega_{\varepsilon}} \prod_{i=1}^{n-1}\left(1+\left\|\left[\kappa_{k, i}\right]_{-}\right\|_{\mathcal{C}\left(\partial \Omega_{k}\right)} \operatorname{diam} \Omega_{k}\right) \tau_{k}(x) \quad \forall x \in \Omega_{\varepsilon},
$$

that allows to estimate $v_{k}$ on any neighborhood of $\bar{\Sigma}$.

\section{Uniqueness}

In this section we will prove the following uniqueness result.

Theorem 4.1 If $(u, v)$ is a solution of system (1)-(2), in the sense of Theorem 3.1, then $v$ is given by (10) and $u \equiv d$ in $\Omega_{v_{f}}:=\left\{x \in \Omega: v_{f}(x)>0\right\}$.

The techniques used in this section come essentially from three papers, $[4,12,21]$. In particular, functional $\Phi$ below is the "stationary" version of the Lagrangian $L$ introduced by Prigozhin in [21] in order to study the evolving shape of a sandpile. The idea of linking solutions of system (1)-(2) and saddle points of $\Phi$ also comes from his work. Moreover, Proposition 4.5 is the generalization to the $n$-dimensional case of the representation formula given in the plane by the first two authors of this paper in [4]. Finally, Proposition 4.6 is a modification of ([12] Proposition 7.1, step 6.); actually, Evans and Gangbo prove there the vanishing property of the transport density $a$ at the ends of transport rays, which is the analogue of the vanishing of our $v_{f}$ on $\bar{\Sigma}$ in the different framework of the Monge-Kantorovich mass transfer problem.

In order to prove Theorem 4.1, let us start by considering the lower semicontinuous functional $\Phi: H_{0}^{1}(\Omega) \times L_{+}^{2}(\Omega) \rightarrow \mathbb{R} \cup\{\infty\}$ defined by

$$
\Phi(w, r)=-\int_{\Omega} f(x) w(x) \mathrm{d} x+\int_{\Omega} \frac{r(x)}{2}\left(|D w(x)|^{2}-1\right) \mathrm{d} x .
$$

We will first prove the uniqueness of the first component of the solution of system (1)-(2). More precisely, we will show that if $(u, v)$ is a solution of system (1)-(2), then $u \equiv d$ in $\Omega_{v_{f}}:=\left\{x \in \Omega: v_{f}(x)>0\right\}$.

Lemma 4.2 If $(u, v)$ is a solution of system (1)-(2), then $(u, v)$ is a saddle point of $\Phi$, in the sense that

$$
\Phi(u, r) \leq \Phi(u, v) \leq \Phi(w, v) \quad \forall(w, r) \in H_{0}^{1}(\Omega) \times L_{+}^{2}(\Omega) .
$$

Proof Since $(u, v)$ is a solution of (1)-(2), then

$$
\int_{\Omega} \frac{v(x)}{2}\left(|D u(x)|^{2}-1\right) \mathrm{d} x=0
$$

and

$$
\int_{\Omega} \frac{r(x)}{2}\left(|D u(x)|^{2}-1\right) \mathrm{d} x \leq 0, \quad \forall r \in L_{+}^{2}(\Omega)
$$


Hence, for any $r \in L_{+}^{2}(\Omega)$ we have

$$
\begin{aligned}
& \Phi(u, v)=-\int_{\Omega} f(x) u(x) \mathrm{d} x \geq \\
& \quad-\int_{\Omega} f(x) u(x) \mathrm{d} x+\int_{\Omega} \frac{r(x)}{2}\left(|D u(x)|^{2}-1\right) \mathrm{d} x=\Phi(u, r) .
\end{aligned}
$$

Moreover, for any $w \in H_{0}^{1}(\Omega)$ we have $\int_{\Omega} \frac{v(x)}{2}|D w(x)-D u(x)|^{2} \mathrm{~d} x \geq 0$ and

$$
-\int_{\Omega} f(x)(w(x)-u(x)) \mathrm{d} x+\int_{\Omega} v(x)\langle D u(x), D w(x)-D u(x)\rangle \mathrm{d} x=0
$$

as a consequence of the fact that for every $\phi \in C_{c}^{\infty}(\Omega)$ (actually for every $\phi \in$ $H_{0}^{1}(\Omega)$, including the case $\left.\phi:=w-u\right)$,

$$
\int_{\Omega} v(x)\langle D u(x), D \phi(x)\rangle \mathrm{d} x=\int_{\Omega} f(x) \phi(x) \mathrm{d} x .
$$

Thus, for any $w \in H_{0}^{1}(\Omega)$,

$$
\begin{aligned}
& \Phi(w, v)=\Phi(u, v)-\int_{\Omega} f(x)(w(x)-u(x)) \mathrm{d} x \\
& +\int_{\Omega} v(x)\langle D u(x), D w(x)-D u(x)\rangle \mathrm{d} x+\int_{\Omega} \frac{v(x)}{2}|D w(x)-D u(x)|^{2} \mathrm{~d} x \\
& \geq \Phi(u, v) .
\end{aligned}
$$

Collecting together (19) and (20) we get the conclusion.

Lemma 4.3 If $(u, v)$ is a solution of system (1)-(2), then also $(d, v)$ is a solution of (1)-(2).

Proof First of all, we claim that $u \equiv d$ in the set $\operatorname{spt}(f)$ as a consequence of Lemma 4.2. In fact, if we consider the set of functions

$$
\mathbb{K}:=\left\{w \in W_{0}^{1, \infty}(\Omega) \mid \quad\|D w\|_{\infty} \leq 1\right\},
$$

then for any $w \in \mathbb{K}$ we have

$$
\int_{\Omega} f(x) w(x) \mathrm{d} x \leq \int_{\Omega} f(x) u(x) \mathrm{d} x
$$

because

$$
\begin{aligned}
& -\int_{\Omega} f(x) w(x) \mathrm{d} x \geq-\int_{\Omega} f(x) w(x) \mathrm{d} x+\int_{\Omega} \frac{v(x)}{2}\left(|D w(x)|^{2}-1\right) \mathrm{d} x \\
& =\Phi(w, v) \geq \Phi(u, v)=-\int_{\Omega} f(x) u(x) \mathrm{d} x .
\end{aligned}
$$

On the other hand it is well known that $d \in \mathbb{K}$ is the largest element of $\mathbb{K}$, meaning that $w \leq d$ for any $w \in \mathbb{K}$. Since $f \geq 0$, the maximality of $d$ implies that $\int_{\Omega} f(x) \bar{u}(x) \mathrm{d} x \leq \int_{\Omega} f(x) d(x) \mathrm{d} x$. Thus $\int_{\Omega} f(x) u(x) \mathrm{d} x=\int_{\Omega} f(x) d(x) \mathrm{d} x$, 
yielding $u \equiv d$ in the set $\operatorname{spt}(f)$. As an easy consequence of the previous equality we also get that $(d, v)$ is a saddle point of functional $\Phi$. Indeed, the coincidence of the two functions on $\operatorname{spt}(f)$ gives $\Phi(d, v)=\Phi(u, v)$ and then for any $w \in H_{0}^{1}(\Omega)$ we have

$$
\Phi(d, v)=\Phi(u, v) \leq \Phi(w, v) .
$$

Moreover, for any choice of $r \in L_{+}^{2}(\Omega), \int_{\Omega} \frac{r(x)}{2}\left(|D d(x)|^{2}-1\right) \mathrm{d} x=0$; therefore

$$
\Phi(d, r)=-\int_{\Omega} f(x) d(x) \mathrm{d} x=\Phi(d, v) .
$$

Now, let us conclude the proof. Consider any $\phi \in C_{c}^{\infty}(\Omega)$. Since $(d, v)$ is a saddle point of $\Phi$, then for any $h>0$

$$
\begin{aligned}
\Phi(d, v) \leq & \Phi(d+h \phi, v) \\
= & -\int_{\Omega} f(x)(d(x)+h \phi(x)) \mathrm{d} x+\int_{\Omega} \frac{v(x)}{2}\left(|D(d+h \phi)(x)|^{2}-1\right) \mathrm{d} x \\
= & \Phi(d, v)+h\left(-\int_{\Omega} f(x) \phi(x) \mathrm{d} x+\int_{\Omega} v(x)\langle D d(x), D \phi(x)\rangle \mathrm{d} x\right) \\
& +\frac{h^{2}}{2} \int_{\Omega} v(x)|D \phi(x)|^{2} \mathrm{~d} x,
\end{aligned}
$$

which gives

$$
\begin{aligned}
& h\left(-\int_{\Omega} f(x) \phi(x) \mathrm{d} x+\int_{\Omega} v(x)\langle D d(x), D \phi(x)\rangle \mathrm{d} x\right) \\
& +\frac{h^{2}}{2} \int_{\Omega} v(x)|D \phi(x)|^{2} \mathrm{~d} x \geq 0 .
\end{aligned}
$$

Dividing by $h$ and letting $h \rightarrow 0^{+}$we obtain

$$
-\int_{\Omega} f(x) \phi(x) \mathrm{d} x+\int_{\Omega} v(x)\langle D d(x), D \phi(x)\rangle \mathrm{d} x \geq 0 .
$$

Replacing $\phi$ by $-\phi$ we also get the opposite inequality.

Proposition 4.4 If $(u, v)$ is a solution of system (1)-(2), then $u \equiv d$ in the set $\left\{x \in \Omega \mid v_{f}(x)>0\right\}$, where $v_{f}$ is the function defined by (10).

Proof By definition of $v_{f}$, it is readily seen that

$$
\begin{aligned}
\operatorname{spt}\left(v_{f}\right) & :=\overline{\left\{x \in \Omega \mid v_{f}(x)>0\right\}} \\
& =\left\{x \in \bar{\Omega} \mid \exists p \in D^{*} d(x) \text { s.t. }[x, x+\tau(x) p] \cap \operatorname{spt}(f) \neq \emptyset\right\} .
\end{aligned}
$$

Hence, for any $y \in\left\{x \in \Omega \mid v_{f}(x)>0\right\}$ we can find $x \in \operatorname{spt}(f)$ such that $d(y)=$ $d(x)-|x-y|$. Now, $u \equiv d$ in $\operatorname{spt}(f)$ —as shown in the proof of Lemma 4.3$u$ is 1-Lipschitz continuous and $d$ is the unique viscosity solution of the eikonal equation with Dirichlet boundary conditions, that is the largest function such that $\|D u\|_{\infty, \Omega} \leq 1$ and $u=0$ on $\partial \Omega$. Therefore we conclude

$$
d(y)=d(x)-|x-y|=u(x)-|x-y| \leq u(y) \leq d(y),
$$

i.e. $u(y)=d(y)$. 
Now that we have proven the uniqueness of the first component of the solution of system (1)-(2), it remains to prove the uniqueness of the second one. In order to do so, we will first exhibit for such a function a representation formula on the set $\Omega \backslash \bar{\Sigma}$ and then analyze its behaviour on $\bar{\Sigma}$.

Proposition 4.5 If $(d, v)$ is a solution of system (1)-(2), then for any $z_{0} \in \Omega \backslash \bar{\Sigma}$ and $\theta \in\left(0, \tau\left(z_{0}\right)\right)$ we have

$$
\begin{gathered}
v\left(z_{0}\right)-\prod_{i=1}^{n-1} \frac{1-\left(d\left(z_{0}\right)+\theta\right) \kappa_{i}\left(z_{0}\right)}{1-d\left(z_{0}\right) \kappa_{i}\left(z_{0}\right)} v\left(z_{0}+\theta D d\left(z_{0}\right)\right) \\
=\int_{0}^{\theta} f\left(z_{0}+t D d\left(z_{0}\right)\right) \prod_{i=1}^{n-1} \frac{1-\left(d\left(z_{0}\right)+t\right) \kappa_{i}\left(z_{0}\right)}{1-d\left(z_{0}\right) \kappa_{i}\left(z_{0}\right)} \mathrm{d} t
\end{gathered}
$$

Proof Set $e_{n}=\operatorname{Dd}\left(z_{0}\right)$ and choose $e_{1}, \ldots, e_{n-1}$ such that $\left\{e_{1}, \ldots, e_{n}\right\}$ is a positively oriented orthonormal basis of $\mathbb{R}^{n}$ and, for any $i=1, \ldots, n-1$, the vector $e_{i}$ is a principal direction whose corresponding principal curvature is $\kappa_{i}\left(z_{0}\right)$. Moreover, let $x_{0}=z_{0}+\theta D d\left(z_{0}\right)$, with $\theta \in\left(0, \tau\left(z_{0}\right)\right)$, and fix $r>0$ sufficiently small such that $S_{0}(r):=\left\{y \in \mathbb{R}^{n}|| y-x_{0} \mid \leq r,\left\langle y-x_{0}, e_{n}\right\rangle=0\right\} \subset \Omega \backslash \bar{\Sigma}$ and for any $y \in S_{0}(r)$ we have $\left\langle D d(y), e_{n}\right\rangle>0$. Finally, denote by $S_{i}(r), i=1,2$, the sets

$$
\begin{aligned}
& S_{1}(r):=\left\{y-\frac{\theta D d(y)}{\left\langle D d(y), e_{n}\right\rangle} \mid y \in S_{0}(r)\right\} \\
& S_{2}(r):=\left\{y-\frac{t D d(y)}{\left\langle D d(y), e_{n}\right\rangle}\left|y-x_{0}\right|=r,\left\langle y-x_{0}, e_{n}\right\rangle=0, \quad t \in[0, \theta]\right\} .
\end{aligned}
$$

and let $D(r)$ be the set enclosed by $S_{0}(r) \cup S_{1}(r) \cup S_{2}(r)$. So,

$$
D(r)=\left\{y-\frac{t D d(y)}{\left\langle D d(y), e_{n}\right\rangle} \mid y \in S_{0}(r), t \in[0, \theta]\right\} \subset \Omega \backslash \bar{\Sigma}
$$

is set with piecewise regular boundary, because of the regularity of $d$. For $\varepsilon>0$, let us define the function

$$
\varphi_{\varepsilon}(x)=\psi_{\varepsilon}\left(d_{r}(x)\right), \quad x \in \Omega,
$$

where $\psi_{\varepsilon}(t)=0$ if $t \leq 0, \psi_{\varepsilon}(t)=t / \varepsilon$ if $0<t<\varepsilon, \psi_{\varepsilon}(t)=1$ if $t \geq \varepsilon$, and $d_{r}$ is the signed distance function from $\partial D(r)$. Choosing $\varphi_{\varepsilon}$ as test function and letting $\varepsilon \rightarrow 0^{+}$, we ontain

$$
\int_{D(r)} f(x) \mathrm{d} x=-\int_{\partial D(r)} v(x)\langle D d(x), v(x)\rangle \mathrm{d} \mathcal{H}^{\mathrm{n}-1}(\mathrm{x}),
$$

where $v(x)$ is the outward unit normal to $\partial D(r)$. Now,

$$
\begin{aligned}
& \int_{\partial D(r)} v(x)\langle D d(x), v(x)\rangle \mathrm{d} \mathcal{H}^{n-1}(x) \\
& \quad=\int_{S_{0}(r) \cup S_{1}(r) \cup S_{2}(r)} v(x)\langle D d(x), v(x)\rangle \mathrm{d} \mathcal{H}^{n-1}(x) \\
& =\int_{S_{0}(r)} v(x)\langle D d(x), v(x)\rangle \mathrm{d} \mathcal{H}^{n-1}(x) \\
& \quad+\int_{S_{1}(r)} v(x)\langle D d(x), v(x)\rangle \mathrm{d} \mathcal{H}^{n-1}(x)
\end{aligned}
$$


because by construction $v(x)$ is orthogonal to $\operatorname{Dd}(x)$ on $S_{2}(r)$. Moreover,

$$
\begin{aligned}
& \int_{S_{0}(r)} v(x)\langle D d(x), v(x)\rangle \mathrm{d} \mathcal{H}^{n-1}(x) \\
& =\int_{S_{0}(r)} v(x)\left\langle D d(x), e_{n}\right\rangle \mathrm{d} \mathcal{H}^{n-1}(x) .
\end{aligned}
$$

Since $D d$ and $v$ are continuous functions in $S_{0}(r)$ and $D d(x) \rightarrow e_{n}$ as $x \rightarrow x_{0}$, then

$$
\lim _{r \rightarrow 0} \frac{1}{\omega_{n-1} r^{n-1}} \int_{S_{0}(r)} v(x)\left\langle D d(x), e_{n}\right\rangle \mathrm{d} \mathcal{H}^{n-1}(x)=v\left(x_{0}\right),
$$

where $\omega_{n-1}$ is the area of the unit ball in $\mathbb{R}^{n-1}$. On the other hand,

$$
\begin{aligned}
& \int_{S_{1}(r)} v(x)\langle D d(x), v(x)\rangle \mathrm{d} \mathcal{H}^{n-1}(x) \\
& =-\int_{S_{0}(r)} v(g(x))\left\langle D d(g(x)), e_{n}\right\rangle|J g(x)| \mathrm{d} \mathcal{H}^{n-1}(x),
\end{aligned}
$$

where

$$
g(x)=x-\frac{\theta D d(x)}{\left\langle\operatorname{Dd}(x), e_{n}\right\rangle}, \quad x \in S_{0}(r)
$$

and $|J g(x)|$ is the modulus of the determinant of the Jacobian matrix of $g$

$$
J g(x)=I+\frac{\theta}{\left\langle D d(x), e_{n}\right\rangle} \sum_{i=1}^{n-1} \frac{\kappa_{i}(x)}{1-d(x) \kappa_{i}(x)} e_{i}(x) \otimes e_{i}(x) .
$$

Since $\lim _{x \rightarrow x_{0}} g(x)=g\left(x_{0}\right)=x_{0}-\theta e_{n}=z_{0}$ and

$$
\lim _{x \rightarrow x_{0}} J g(x)=I+\sum_{i=1}^{n-1} \frac{\theta \kappa_{i}\left(z_{0}\right)}{1-d\left(z_{0}\right) \kappa_{i}\left(z_{0}\right)} e_{i} \otimes e_{i},
$$

we have

$$
\lim _{x \rightarrow x_{0}}|J g(x)|=\prod_{i=1}^{n-1}\left(1+\frac{\theta \kappa_{i}\left(z_{0}\right)}{1-d\left(z_{0}\right) \kappa_{i}\left(z_{0}\right)}\right),
$$

and then we conclude

$$
\begin{gathered}
\lim _{r \rightarrow 0} \frac{1}{\omega_{n-1} r^{n-1}} \int_{S_{1}(r)} v(x)\langle D d(x), v(x)\rangle \mathrm{d} \mathcal{H}^{n-1}(x) \\
=-v\left(z_{0}\right) \prod_{i=1}^{n-1}\left(1+\frac{\theta \kappa_{i}\left(z_{0}\right)}{1-d\left(z_{0}\right) \kappa_{i}\left(z_{0}\right)}\right) .
\end{gathered}
$$

So now it only remains to estimate $\lim _{r \rightarrow 0} \frac{1}{\omega_{n-1} r^{n-1}} \int_{D(r)} f(x) \mathrm{d} x$. Exploiting the structure of the set $D(r)$, it is easy to see that we can write

$$
\int_{D(r)} f(x) \mathrm{d} x=\int_{0}^{\theta} \mathrm{d} t \int_{S_{t}(r)} f(z) \mathrm{d} \mathcal{H}^{\mathrm{n}-1}(\mathrm{z})
$$


where

$$
S_{t}(r):=\left\{y-\frac{t}{\left\langle D d(y), e_{n}\right\rangle} D d(y) \mid y \in S_{1}(r)\right\} .
$$

Hence, using the previous computations and the continuity of $f$ we finally find

$$
\begin{aligned}
& \lim _{r \rightarrow 0} \frac{1}{\omega_{n-1} r^{n-1}} \int_{D(r)} f(x) \mathrm{d} x \\
& \quad=\int_{0}^{\theta} f\left(x_{0}-t D d\left(x_{0}\right)\right) \prod_{i=1}^{n-1}\left(1+\frac{t \kappa_{i}\left(x_{0}\right)}{1-d\left(x_{0}\right) \kappa_{i}\left(x_{0}\right)}\right) \mathrm{d} t .
\end{aligned}
$$

Collecting together (25), (27) and (29) and recalling identity (23), we can write

$$
\begin{gathered}
\int_{0}^{\theta} f\left(x_{0}-t D d\left(x_{0}\right)\right) \prod_{i=1}^{n-1}\left(1+\frac{t \kappa_{i}\left(x_{0}\right)}{1-d\left(x_{0}\right) \kappa_{i}\left(x_{0}\right)}\right) \mathrm{d} t \\
=-v\left(x_{0}\right)+v\left(z_{0}\right) \prod_{i=1}^{n-1}\left(1+\frac{\theta \kappa_{i}\left(z_{0}\right)}{1-d\left(z_{0}\right) \kappa_{i}\left(z_{0}\right)}\right) .
\end{gathered}
$$

In order to represent (30) in the form (22) we only have to divide both sides of (30) by

$$
\prod_{i=1}^{n-1}\left(1+\frac{\theta \kappa_{i}\left(x_{0}\right)}{1-d\left(x_{0}\right) \kappa_{i}\left(x_{0}\right)}\right)
$$

and make a change of variable in the right-hand integral. Indeed, recalling that $x_{0}=z_{0}+\theta D d\left(z_{0}\right), D d\left(x_{0}\right)=D d\left(z_{0}\right)$ and $\kappa_{i}\left(x_{0}\right)=\kappa_{i}\left(z_{0}\right)$, the above computation gives

$$
\begin{gathered}
\int_{0}^{\theta} f\left(z_{0}+(\theta-t) D d\left(z_{0}\right)\right) \prod_{i=1}^{n-1}\left(\frac{1-\left(d\left(z_{0}\right)+\theta-t\right) \kappa_{i}\left(z_{0}\right)}{1-d\left(z_{0}\right) \kappa_{i}\left(z_{0}\right)}\right) \mathrm{d} t \\
\quad=-v\left(z_{0}+\theta D d\left(z_{0}\right)\right)+v\left(z_{0}\right) \prod_{i=1}^{n-1}\left(\frac{1-\left(d\left(z_{0}\right)+\theta\right) \kappa_{i}\left(z_{0}\right)}{1-d\left(z_{0}\right) \kappa_{i}\left(z_{0}\right)}\right) .
\end{gathered}
$$

Now the representation formula (22) follows as soon as we replace the variable $t$ by $\theta-s$ in the above right-hand integral.

Proposition 4.6 If $(d, v)$ is a solution of system (1)-(2), then $v \equiv 0$ on $\bar{\Sigma}$.

Proof Since $v$ is a continuous function, it suffices to prove that $v \equiv 0$ on $\Sigma$. So, let us fix any $x_{0} \in \Sigma$ and choose $\varepsilon>0$ sufficiently small such that $B_{\varepsilon}\left(x_{0}\right) \subset \Omega$. Then, for any $x \in B_{1}(0)$ set

$$
\left\{\begin{array}{l}
d_{\varepsilon}(x):=\frac{d\left(x_{0}+\varepsilon x\right)-d\left(x_{0}\right)}{\varepsilon} \\
v_{\varepsilon}(x):=v\left(x_{0}+\varepsilon x\right), \quad f_{\varepsilon}(x):=f\left(x_{0}+\varepsilon x\right) .
\end{array}\right.
$$


By construction, for any $\varepsilon>0$ as above $d_{\varepsilon}(0)=0$ and $\left|D d_{\varepsilon}(x)\right|=\mid D d\left(x_{0}+\right.$ $\varepsilon x) \mid=1$ almost everywhere in $B_{1}(0)$. Hence, there exist a sequence $\left\{\varepsilon_{j}\right\}_{j \in \mathbb{N}}$, $\varepsilon_{j} \rightarrow 0^{+}$and a 1 -Lipschitz function $d_{0}: B_{1}(0) \rightarrow \mathbb{R}$ such that $d_{\varepsilon_{j}} \rightarrow d_{0}$ in the uniform topology in $B_{1}(0)$. Moreover, being $\left|D d_{\varepsilon_{j}}(x)\right|=1$ in the viscosity sense in $B_{1}(0)$, by [2, Proposition 2.2] also $\left|D d_{0}(x)\right|=1$ in the viscosity sense in $B_{1}(0)$, which gives $\left|D d_{0}(x)\right|=1$ almost everywhere. Thus,

$$
\lim _{j \rightarrow \infty} \int_{B_{1}(0)}\left|D d_{\varepsilon_{j}}(x)\right|^{2} \mathrm{~d} x=\omega_{n}=\int_{B_{1}(0)}\left|D d_{0}(x)\right|^{2} \mathrm{~d} x,
$$

which implies, together with the uniform convergence of $d_{\varepsilon_{j}}$ to $d_{0}$, the convergence of $D d_{\varepsilon_{j}}$ to $D d_{0}$ in the $L^{2}$ topology.

Also, $D^{2} d_{\varepsilon_{j}}(x)=\varepsilon_{j} D^{2} d\left(x_{0}+\varepsilon_{j} x\right) \leq \varepsilon_{j} C$ in the sense of distributions, because the function $d$ is a semiconcave function with linear modulus in $B_{\varepsilon}\left(x_{0}\right) \subset$ $\Omega$. Therefore, $d_{0}$ is a concave function. Finally, the functions $v_{\varepsilon_{j}}$ and $f_{\varepsilon_{j}}$ defined above uniformly converge to $v\left(x_{0}\right)$ and $f\left(x_{0}\right)$ respectively and the pair $\left(d_{\varepsilon_{j}}, v_{\varepsilon_{j}}\right)$ solves

$$
-\operatorname{div}\left(v_{\varepsilon_{j}} D d_{\varepsilon_{j}}\right)=\varepsilon_{j} f_{\varepsilon_{j}} \quad \text { in } B_{1}(0)
$$

in the weak sense, because $(d, v)$ solves (1)-(2). Passing to the limit as $j \rightarrow \infty$ we then obtain that $d_{0}$ is a weak solution of

$$
-\operatorname{div}\left(v\left(x_{0}\right) D d_{0}(x)\right)=0 \quad x \in B_{1}(0) .
$$

Now, if $v\left(x_{0}\right) \neq 0$, the previous equation turns out to be the classical Laplace equation

$$
\triangle d_{0}=0 \quad \text { in } B_{1}(0)
$$

and it is well-known that any weak Lipschitz solution in the ball of this equation is actually analytic. On the other hand, $d_{0}$ cannot be differentiable in $x=0$, because $d_{0}$ is the 'blow up' of the distance function around a singular point $x_{0}$. Hence $v\left(x_{0}\right)=0$ and the proof is complete.

The last two propositions allow us to prove Theorem 4.1 as a simple corollary. Indeed, we already know by Proposition 4.4 that if $(u, v)$ is a solution of system (1)-(2), then $u \equiv d$ on the set $\Omega_{v_{f}}=\left\{v_{f}>0\right\}$. So it only remains to prove that $v \equiv v_{f}$ in $\Omega$, where $v_{f}$ is given by (10). But Proposition 4.6 guarantees that $v \equiv 0$ in $\bar{\Sigma}$, while Proposition 4.5 tells us that for any $z_{0} \in \Omega \backslash \bar{\Sigma}$ and $\theta \in\left(0, \tau\left(z_{0}\right)\right)$

$$
\begin{aligned}
& v\left(z_{0}\right)-\prod_{i=1}^{n-1} \frac{1-\left(d\left(z_{0}\right)+\theta\right) \kappa_{i}\left(z_{0}\right)}{1-d\left(z_{0}\right) \kappa_{i}\left(z_{0}\right)} v\left(z_{0}+\theta D d\left(z_{0}\right)\right) \\
& =\int_{0}^{\theta} f\left(z_{0}+t D d\left(z_{0}\right)\right) \prod_{i=1}^{n-1} \frac{1-\left(d\left(z_{0}\right)+t\right) \kappa_{i}\left(z_{0}\right)}{1-d\left(z_{0}\right) \kappa_{i}\left(z_{0}\right)} d t .
\end{aligned}
$$

Hence, letting $\theta \rightarrow \tau\left(z_{0}\right)^{-}$and using the continuity of $v$ we obtain the coincidence of $v$ and $v_{f}$ at the point $z_{0}$. 


\section{Minimization of integral functionals}

In this section we shall prove the existence of minimizers in $W_{0}^{1,1}(\Omega)$ for the integral functional

$$
J_{0}(u)=\int_{\Omega}[h(|D u|)-f(x) u] \mathrm{d} x, \quad u \in W_{0}^{1,1}(\Omega),
$$

where $f \in L^{\infty}(\Omega)$ is a nonnegative function and $h:[0,+\infty) \rightarrow[0,+\infty]$ is a lower semicontinuous function (possibly with extended values) satisfying

$$
h(R)=0, \quad h(s) \geq \max \{0, \Lambda(s-R)\} \quad \text { for some constants } R, \Lambda>0 .
$$

More precisely, under suitable assumptions on the domain $\Omega$, the function $u_{\Omega}(x)=R d(x), x \in \Omega$, is a minimizer of $J_{0}$. As remarked in the Introduction, the set $\Omega$ need not to be convex, as required in previous results (see [8, 24]). The main assumption on $\Omega$ is that it can be approximated, in the Hausdorff metric, by smooth domains satisfying a suitable uniform bound on the mean curvature of their boundaries (see Definition 5.1 below). We shall show in Proposition 5.2 that such class of domains contains Federer's sets with positive reach (see [14]).

The link with the system of PDE (1)-(2) is that (11) is the Euler-Lagrange equation associated to the integral functional $J_{0}$ in $u_{\Omega}$, provided that the solution $v_{f}$ satisfies a suitable upper bound. This bound can be obtained using the explicit representation (10) of $v_{f}$ and the bounds on the mean curvature of $\partial \Omega$ (see Proposition 5.9 below).

Definition 5.1 We say that a set $\Omega$ is a smooth $K$-admissible domain, $K \in \mathbb{R}$, if it is a connected open bounded subset of $\mathbb{R}^{n}$ with $C^{2}$ boundary, such that the mean curvature of $\partial \Omega$ is bounded below by $K$, that is

$$
H(y):=\frac{1}{n-1} \sum_{i=1}^{n-1} \kappa_{i}(y) \geq K \quad \forall y \in \partial \Omega .
$$

A set $\Omega \subset \mathbb{R}^{n}$ is a $K$-admissible domain if it is a connected open bounded subset of $\mathbb{R}^{n}$ and there exists a sequence $\left(\Omega_{j}\right)_{j}$, with $\Omega_{j}$ smooth $K_{j}$-admissible domain, such that $\lim _{j} K_{j}=K$ and the Hausdorff distance $d_{H}\left(\Omega_{j}, \Omega\right)$ tends to 0 as $j \rightarrow$ $+\infty$.

We remark that every connected bounded open set $\Omega \subset \mathbb{R}^{n}$ with $C^{2}$ boundary is a $K$-admissible smooth domain for every $K$ satisfying

$$
K \leq \min _{y \in \partial \Omega} H(y) .
$$

Furthermore, it is readily seen that every convex domain $\Omega \subset \mathbb{R}^{n}$ is a $K$ admissible domain, for every $K \leq 0$. Namely, every convex domain can be approximated, in the Hausdorff topology, by smooth $\left(C^{\infty}\right)$ convex domains (see [22] Sect. 3.3). Then, it is enough to observe that a smooth convex domain satisfies $\kappa_{i}(y) \geq 0$ for every $i$ and every $y \in \partial \Omega$.

Another class of possibly nonsmooth and nonconvex admissible domains is given by connected domains satisfying a uniform exterior sphere condition. These sets are also known in literature as sets of positive reach (see [14]). 
Proposition 5.2 Let $\Omega \subset \mathbb{R}^{n}$ be a connected bounded open set satisfying a uniform exterior sphere condition for some radius $R \in(0,+\infty]$. Then $\Omega$ is a $(-16 / R)$-admissible domain.

Proof Let us denote by $d_{\bar{\Omega}}(x), x \in \mathbb{R}^{n}$, the distance of $x$ to $\bar{\Omega}$ and set

$$
\Omega_{R / 2}:=\left\{x \in \mathbb{R}^{n}: d_{\bar{\Omega}}(x)<R / 2\right\} .
$$

We will first prove that $d_{\bar{\Omega}}(x)+\frac{4}{R}|x|^{2}$ is convex locally in $\Omega_{R / 2} \backslash \bar{\Omega}$. It is well known (see for instance [15]) that the exterior sphere condition implies the uniqueness of the projection of any point $x \in \Omega_{R / 2} \backslash \bar{\Omega}$ onto the set $\bar{\Omega}$, so that the gradient $D d_{\bar{\Omega}}(x)$ is well defined. Consider any $x, y \in \Omega_{R / 2} \backslash \bar{\Omega}$. Suppose first that $d_{\bar{\Omega}}(x)=d_{\bar{\Omega}}(y)=: \rho$. By the exterior sphere condition, we have that

$x \notin B\left(y+(R-\rho) D d_{\bar{\Omega}}(y), R-\rho\right) \quad$ and $\quad y \notin B\left(x+(R-\rho) D d_{\bar{\Omega}}(x), R-\rho\right)$.

Hence,

$$
|x-y|^{2}-2\left\langle(R-\rho) D d_{\bar{\Omega}}(y), x-y\right\rangle \geq 0
$$

and

$$
|x-y|^{2}+2\left\langle(R-\rho) D d_{\bar{\Omega}}(x), x-y\right\rangle \geq 0 .
$$

Adding the above inequalities we obtain

$$
\left\langle D d_{\bar{\Omega}}(x)-D d_{\bar{\Omega}}(y), x-y\right\rangle \geq-\frac{1}{R-\rho}|x-y|^{2} \geq-\frac{2}{R}|x-y|^{2} .
$$

Suppose, on the other hand, that $d_{\bar{\Omega}}(x) \neq d_{\bar{\Omega}}(y)$ — say $d_{\bar{\Omega}}(x)>d_{\bar{\Omega}}(y)$ —and consider the point $z:=x-\left(d_{\bar{\Omega}}(x)-d_{\bar{\Omega}}(y)\right) D d_{\bar{\Omega}}(x)$. It is clear that $z \in \Omega_{R / 2}$, $d_{\bar{\Omega}}(z)=d_{\bar{\Omega}}(y)$ and $D d_{\bar{\Omega}}(z)=D d_{\bar{\Omega}}(x)$. Then, by (35), we have

$$
\left\langle D d_{\bar{\Omega}}(x)-D d_{\bar{\Omega}}(y), z-y\right\rangle \geq-\frac{2}{R}|z-y|^{2} .
$$

Writing the previous inequality in terms of $x$ and using the Lipschitz properties of the distance function $d_{\bar{\Omega}}$, we finally obtain

$$
\begin{aligned}
& \left\langle D d_{\bar{\Omega}}(x)-D d_{\bar{\Omega}}(y), x-y\right\rangle \geq\left(d_{\bar{\Omega}}(x)-d_{\bar{\Omega}}(y)\right)\left\langle D d_{\bar{\Omega}}(x)-D d_{\bar{\Omega}}(y), D d_{\bar{\Omega}}(x)\right\rangle \\
& \quad-\frac{2}{R}|x-y|^{2}-\frac{2}{R}\left(d_{\bar{\Omega}}(x)-d_{\bar{\Omega}}(y)\right)^{2}+\frac{4}{R}\left(d_{\bar{\Omega}}(x)-d_{\bar{\Omega}}(y)\right)\left\langle D d_{\bar{\Omega}}(x), x-y\right\rangle \\
& \quad \geq-\frac{8}{R}|x-y|^{2} .
\end{aligned}
$$

This actually proves that $d_{\bar{\Omega}}(x)+\frac{4}{R}|x|^{2}$ is convex locally in $\Omega_{R / 2} \backslash \bar{\Omega}$.

Let us now construct the approximating sequence of $(-16 / R)$-admissible domains. For any $0<\sigma<\frac{R}{8}$ and $0<\varepsilon<\frac{\sigma}{2}$, consider the sets

$$
\Omega_{\sigma}^{\varepsilon}:=\left\{x \in \mathbb{R}^{n}:\left(\rho_{\varepsilon} * b_{\Omega}\right)(x)<\sigma\right\},
$$


where $b_{\Omega}$ is the signed distance $b_{\Omega}(x):=d_{\bar{\Omega}}(x)-d_{\mathbb{R}^{n} \backslash \Omega}(x)$ and $\rho_{\varepsilon}$ is the family of mollifiers defined by

$$
\begin{aligned}
\rho_{\varepsilon}(x) & =\frac{1}{\varepsilon^{n}} \rho\left(\frac{x}{\varepsilon}\right), \\
\rho(y) & := \begin{cases}\left(\int_{B(0,1)} \mathrm{e}^{-\frac{1}{1-|z|^{2}}} d z\right)^{-1} \mathrm{e}^{-\frac{1}{1-|y|^{2}}} & |y| \leq 1 \\
0 & |y|>1 .\end{cases}
\end{aligned}
$$

Note that our choice of $\sigma$ and $\varepsilon$ assures that $\Omega_{\sigma}^{\varepsilon} \subset \Omega_{R / 2}$. Set $d_{\varepsilon}:=\rho_{\varepsilon} * b_{\Omega}$. Then $d_{\varepsilon} \in C^{\infty}\left(\mathbb{R}^{n}\right)$ and $d_{\varepsilon}, D d_{\varepsilon}$ uniformly converge to $b_{\Omega}, D b_{\Omega}$ on any compact subset of $\mathbb{R}^{n}$ and of $\Omega_{R / 2} \backslash \bar{\Omega}$, respectively, as $\varepsilon \rightarrow 0$. This actually gives that

$$
\partial \Omega_{\sigma}^{\varepsilon}:=\left\{x \in \mathbb{R}^{n}:\left(\rho_{\varepsilon} * b_{\Omega}\right)(x)=\sigma\right\}
$$

are $C^{\infty}$ boundaries for any $0<\sigma<R / 8$ and $\varepsilon<\sigma / 2$, converging to $\partial \Omega$ as $\sigma \rightarrow 0$. We also claim that the above conditions on $\sigma$ and $\varepsilon$ guarantee that also $\partial \Omega_{\sigma}^{\varepsilon} \subset \Omega_{R / 2-\varepsilon} \backslash \bar{\Omega}_{\varepsilon}$. Indeed, there exists $\varepsilon(\sigma)$ such that for any $\varepsilon<\varepsilon(\sigma)$ $\left|d_{\varepsilon}(x)-b_{\Omega}(x)\right|<\sigma / 2$, for any $x \in \Omega_{R / 2} \backslash \bar{\Omega}$. Hence, for any $x \in \partial \Omega_{\sigma}^{\varepsilon}$

$$
b_{\Omega}(x)=\left(b_{\Omega}(x)-d_{\varepsilon}(x)\right)+d_{\varepsilon}(x)<\frac{\sigma}{2}+\sigma=\frac{3}{2} \sigma<\frac{R}{2}-\varepsilon
$$

and

$$
b_{\Omega}(x)=d_{\varepsilon}(x)-\left(d_{\varepsilon}(x)-b_{\Omega}(x)\right)>\sigma-\frac{\sigma}{2}=\frac{1}{2} \sigma>\varepsilon,
$$

which means that $b_{\Omega}(x)=d_{\bar{\Omega}}(x)$ and $\varepsilon<d_{\bar{\Omega}}(x)<\frac{R}{2}-\varepsilon$. As a consequence of the inclusion $\partial \Omega_{\sigma}^{\varepsilon} \subset \Omega_{R / 2-\varepsilon} \backslash \bar{\Omega}_{\varepsilon}$ we also obtain a bound from below for $D d_{\varepsilon}$ on $\partial \Omega_{\sigma}^{\varepsilon}$, provided $\sigma$ (and then $\varepsilon$ ) is small enough. Indeed, since $D d_{\varepsilon}$ uniformly converges to $D b_{\Omega}$ on any compact subset of $\Omega_{R / 2} \backslash \bar{\Omega}$ as $\varepsilon \rightarrow 0$, we can take $\sigma$ so small that for $\varepsilon<\min \{\sigma / 2, \varepsilon(\sigma)\}$ we have $\left|D d_{\varepsilon}(x)-D b_{\Omega}(x)\right|<1 / 2$ for any $x \in \partial \Omega_{\sigma}^{\varepsilon}$ and then $\left|D d_{\varepsilon}(x)\right|>1 / 2$ for any $x \in \partial \Omega_{\sigma}^{\varepsilon}$. Let us now prove that the maps $d_{\varepsilon}$ are semiconvex in $\Omega_{R / 2-\varepsilon} \backslash \bar{\Omega}_{\varepsilon}$ with constant $4 / R$. Indeed, since $d_{\bar{\Omega}}(x)+\frac{4}{R}|x|^{2}$ is convex locally on $\Omega_{R / 2} \backslash \bar{\Omega}$, our choice of $\varepsilon$ gives that, in the set $\Omega_{R / 2-\varepsilon} \backslash \bar{\Omega}_{\varepsilon}$,

$$
\rho_{\varepsilon} *\left(b_{\Omega}+\frac{4}{R}|\cdot|^{2}\right)=\rho_{\varepsilon} *\left(d_{\bar{\Omega}}+\frac{4}{R}|\cdot|^{2}\right)=\rho_{\varepsilon} * d_{\bar{\Omega}}+\frac{4}{R} \rho_{\varepsilon} *|\cdot|^{2}
$$

is still convex locally. Being also the map $\rho_{\varepsilon} *\left(d_{\bar{\Omega}}+\frac{4}{R}|\cdot|^{2}\right)$ regular, we deduce

$$
0 \leq D^{2}\left(\rho_{\varepsilon} *\left(d_{\bar{\Omega}}+\frac{4}{R}|\cdot|^{2}\right)\right)=D^{2} d_{\varepsilon}+\frac{8}{R} I,
$$

which is the desired semiconvex property of $d_{\varepsilon}$. In order to complete the proof, it suffices to look at the curvature matrix of $\partial \Omega_{\sigma}^{\varepsilon}$. As a matter of fact, for any $x \in \partial \Omega_{\sigma}^{\varepsilon}$ the outer unit normal of $\partial \Omega_{\sigma}^{\varepsilon}$ at $x$ is given by $\nu_{\sigma}^{\varepsilon}(x)=\frac{\nabla d_{\varepsilon}(x)}{\left|\nabla d_{\varepsilon}(x)\right|}$. Hence, 
the principal curvatures of $\partial \Omega_{\sigma}^{\varepsilon}$ at $x$ are the eigenvalues of the matrix $D\left(\frac{\nabla d_{\varepsilon}(x)}{\left|\nabla d_{\varepsilon}(x)\right|}\right)$ restricted to the subspace $V^{\varepsilon}$ orthogonal to $\nabla d_{\varepsilon}(x)$. But

$$
D\left(\frac{\nabla d_{\varepsilon}(x)}{\left|\nabla d_{\varepsilon}(x)\right|}\right)=\frac{1}{\left|\nabla d_{\varepsilon}(x)\right|} D^{2} d_{\varepsilon}(x)-\frac{1}{\left|\nabla d_{\varepsilon}(x)\right|^{3}} D^{2} d_{\varepsilon}(x) \nabla d_{\varepsilon}(x) \otimes \nabla d_{\varepsilon}(x),
$$

so that

$$
\left.D\left(\frac{\nabla d_{\varepsilon}(x)}{\left|\nabla d_{\varepsilon}(x)\right|}\right)\right|_{V^{\varepsilon}}=\left.\frac{1}{\left|\nabla d_{\varepsilon}(x)\right|} D^{2} d_{\varepsilon}(x)\right|_{V^{\varepsilon}} .
$$

Thus, recalling that $\left|\nabla d_{\varepsilon}(x)\right|>1 / 2$ and using (36), we conclude that any principal curvature of $\partial \Omega_{\sigma}^{\varepsilon}$ at $x$ is bounded from below by $-\frac{16}{R}$, yielding

$$
H_{\sigma}^{\varepsilon}(x) \geq-\frac{16}{R},
$$

for any $x \in \partial \Omega_{\sigma}^{\varepsilon}$.

Our main existence result concerns integral functionals more general than the one defined in (33). In the following, $r_{\Omega}$ will denote the inradius of the set $\Omega$, that is, the supremum of the radii of the balls contained in $\Omega$.

Theorem 5.3 Let $h:[0,+\infty) \rightarrow[0,+\infty]$ be a lower semicontinuous function satisfying (34), let $\Omega \subset \mathbb{R}^{n}$ be a $K$-admissible domain, and let $g: \Omega \times \mathbb{R} \rightarrow \mathbb{R}$ be a measurable function, Lipschitz continuous with respect to the second variable, satisfying

$$
\begin{aligned}
& g(\cdot, 0) \in L^{1}(\Omega), \\
& 0 \leq D_{u} g(x, u) \leq L, \quad \text { a.e. }(x, u) \in \Omega \times \mathbb{R} .
\end{aligned}
$$

If $L \cdot c\left(K, r_{\Omega}\right) \leq \Lambda$, where

$$
c\left(K, r_{\Omega}\right):=\left\{\begin{array}{cl}
\frac{1-\left(1-K r_{\Omega}\right)^{n}}{n K}, & \text { if } K \neq 0, \\
r_{\Omega}, & \text { if } K=0,
\end{array}\right.
$$

then the function $u_{\Omega}(x)=R d(x), x \in \Omega$, is a minimizer of the functional

$$
J(u)=\int_{\Omega}[h(|D u|)-g(x, u)] \mathrm{d} x
$$

in $W_{0}^{1,1}(\Omega)$.

Remark 5.4 If $g$ satisfies $-L \leq D_{u} g(x, u) \leq 0$ for a.e. $(x, u) \in \Omega \times \mathbb{R}$, then Theorem 5.3 still holds with $u_{\Omega}(x)=-R d(x)$.

Remark 5.5 If $\Omega \subset \mathbb{R}^{n}$ is a convex domain, then condition $L c\left(K, r_{\Omega}\right) \leq \Lambda$ is certainly satisfied provided that

$$
L r_{\Omega} \leq \Lambda .
$$

Namely, it is enough to observe that a convex domain is a 0 -admissible domain, and that $c\left(0, r_{\Omega}\right)=r_{\Omega}$. Condition (41) was first introduced in [8] in the case $g(x, u)=u$. In [6] it was proven that, if (41) does not hold, then the functional $J$ need not have minimizers in $W_{0}^{1,1}(\Omega)$. 
Example 5.6 The assumptions of Theorem 5.3 for the existence of a minimizer of $J$ are optimal in the following sense. Let $h(s)=\max \{0, \Lambda(s-R)\}$ for some positive constants $\Lambda$ and $R$, let $g(x, u)=u$ and let $\Omega=B_{r}(0) \subset \mathbb{R}^{n}$. Then $r_{\Omega}=r$, and $\Omega$ is a $(1 / r)$-admissible domain.

Since $c(1 / r, r)=r / n$, Theorem 5.3 states that the function $u_{\Omega}(x)=R d(x)$ is a minimizer to $J$ provided that $r \leq n \Lambda$. This condition is optimal: indeed, we are going to show that the functional $J$ is not even bounded from below if $r>n \Lambda$. Let us define the sequence of functions in $W_{0}^{1,1}(\Omega)$

$$
u_{k}(x)=\left\{\begin{array}{ll}
k(r-|x|), & \text { if } n \Lambda<|x|<r, \\
R(r-|x|), & \text { if }|x| \leq n \Lambda,
\end{array} \quad k \in \mathbb{N} .\right.
$$

A straightforward computation shows that, for $k \geq R$,

$$
J\left(u_{k}\right)=\frac{\omega_{n}}{n+1}[\psi(n \Lambda)-\psi(r)] k+A,
$$

where $\omega_{n}$ is the $n$-dimensional Lebesgue measure of the unit ball of $\mathbb{R}^{n}, A$ is a constant independent of $k$, and $\psi(\rho)=\rho^{n+1}-(n+1) \Lambda \rho^{n}$. Since the function $\psi$ is strictly increasing for $\rho \geq n \Lambda$, and $r>n \Lambda$, we have that $\psi(n \Lambda)-\psi(r)<0$, hence $\lim _{k \rightarrow+\infty} J\left(u_{k}\right)=-\infty$.

The remaining part of this section will be devoted to the proof of Theorem 5.3.

The relation between the existence of solutions to (1)-(2), given by Theorem 3.1, and the existence of minimizers of $J$, can be better understood starting from the following particular case of Theorem 5.3.

Proposition 5.7 Let $h:[0,+\infty) \rightarrow[0,+\infty]$ be a lower semicontinuous function satisfying (34), let $\Omega \subset \mathbb{R}^{n}$ be a smooth $K$-admissible domain, and let $f \in L^{\infty}(\Omega)$ be a nonnegative Lipschitz continuous function.

If $\|f\|_{\infty, \Omega} c\left(K, r_{\Omega}\right) \leq \Lambda$, then the function $u_{\Omega}(x)=R d(x)$ is a minimizer in $W_{0}^{1,1}(\Omega)$ of the functional $J_{0}$ defined in (33).

Proof Let $v_{f}$ be the continuous function defined in (10). We claim that the following bound on $v_{f}$ holds true:

$$
0 \leq v_{f}(x) \leq\|f\|_{\infty, \Omega} c\left(K, r_{\Omega}\right), \quad \forall x \in \Omega .
$$

In order not to interrupt the main flow of the arguments, we postpone the proof of (42) to Proposition 5.9 below.

By assumption, we clearly have

$$
0 \leq v_{f}(x) \leq \Lambda, \forall x \in \Omega .
$$

Let $u \in W_{0}^{1,1}(\Omega)$. Since $h$ satisfies (34) and $v_{f}$ satisfies (43), we have that

$$
\begin{aligned}
h(|D u(x)|) & \geq v_{f}(x)(|D u(x)|-R) \\
& \geq h\left(\left|D u_{\Omega}(x)\right|\right)+v_{f}(x)\left\langle D d(x), D u(x)-D u_{\Omega}(x)\right\rangle,
\end{aligned}
$$

hence

$$
J_{0}(u) \geq J_{0}\left(u_{\Omega}\right)+\delta
$$


where

$$
\delta=\int_{\Omega}\left[v_{f}(x)\left\langle D d(x), D u(x)-D u_{\Omega}(x)\right\rangle-f(x)\left(u(x)-u_{\Omega}(x)\right)\right] \mathrm{d} x .
$$

Since $v_{f}$ is bounded, by a density argument the equation (11) holds for every $\phi \in W_{0}^{1,1}(\Omega)$. Choosing $\phi=u-u_{\Omega}$, we obtain that $\delta$ vanishes, so that $J_{0}(u) \geq$ $J_{0}\left(u_{\Omega}\right)$. Since $u$ was an arbitrary function in $W_{0}^{1,1}(\Omega)$, we have proven that $u_{\Omega}$ is a minimizer of $J_{0}$ in $W_{0}^{1,1}(\Omega)$.

The estimate (42) used in the proof of Proposition 5.7 is an easy consequence of the following lemma.

Lemma 5.8 Let $v:[0, r] \rightarrow \mathbb{R}$ be the function defined by

$$
v(t):=\int_{t}^{r} \prod_{i=1}^{n-1} \frac{1-s \kappa_{i}}{1-t \kappa_{i}} \mathrm{~d} s
$$

where $\left(\kappa_{1}, \ldots, \kappa_{n-1}\right)$ belongs to the set

$$
C:=\left\{\left(\sigma_{1}, \ldots, \sigma_{n-1}\right) \in \mathbb{R}^{n-1} ; \sigma_{i} \leq \frac{1}{r} \forall i, \sum_{j=1}^{n-1} \sigma_{j} \geq(n-1) K\right\}
$$

for some constant $K \leq 1 / r$. Then, for every $t \in[0, r]$,

$$
0 \leq v(t) \leq v_{0}(t):= \begin{cases}\frac{(1-t K)^{n}-(1-r K)^{n}}{n K(1-t K)^{n-1}}, & \text { if } K \neq 0, \\ r-t, & \text { if } K=0 .\end{cases}
$$

As a consequence, $v(t) \leq c(K, r)$ for every $t \in[0, r]$, where $c(K, r)$ is the constant defined in (39).

Proof Since $K \leq 1 / r$, the closed set $C$ is not empty. Moreover, given a point $\left(\sigma_{1}, \ldots, \sigma_{n-1}\right) \in C$, for every $i$ one has

$$
\frac{1}{r} \geq \sigma_{i}=\sum_{j} \sigma_{j}-\sum_{j \neq i} \sigma_{j} \geq(n-1) K-\frac{n-2}{r},
$$

so that $C$ is a nonempty compact subset of $\mathbb{R}^{n-1}$.

Let us consider the function $\varphi^{t}: C \rightarrow \mathbb{R}$ defined by

$$
\varphi^{t}\left(\sigma_{1}, \ldots, \sigma_{n-1}\right)=\sum_{j=1}^{n-1} \frac{\sigma_{j}}{1-t \sigma_{j}}
$$

where $t \in[0, r)$ plays the role of a parameter. Since $\frac{\partial \varphi^{t}}{\partial \sigma_{j}}=\left(1-t \sigma_{j}\right)^{-2}>0$, $\varphi^{t}$ does not have critical points in the interior of $C$. Moreover, it is clear that $\varphi^{t}$ attains its minimum on the constraint

$$
\sum_{j} \sigma_{j}=(n-1) K
$$


Using the Lagrange multipliers rule we get that the minimum is attained at the point $\sigma_{1}=\cdots=\sigma_{n-1}=K$, hence

$$
\min _{C} \varphi^{t}=\frac{(n-1) K}{1-t K} .
$$

The function $v:[0, r] \rightarrow \mathbb{R}$, defined in (44), is differentiable in $[0, r)$. Moreover, $0 \leq v(t) \leq \int_{t}^{r}\left(1+r[k]_{\infty}^{-}\right)^{n-1} \mathrm{~d} s=\left(1+r[k]_{\infty}^{-}\right)^{n-1}(r-t)$, where

$$
[k]_{\infty}^{-}:=\max \left\{\left[k_{i}\right]^{-}: i=\{1, \ldots, n-1\},\left(k_{1}, \ldots, k_{n-1}\right) \in C\right\} .
$$

Hence $\lim _{t \rightarrow r} v(t)=0=v(r)$. Let us compute the derivative of $v$ : for every $t \in[0, r)$ we have

$$
v^{\prime}(t)=-1+\left(\sum_{j=1}^{n-1} \frac{\kappa_{j}}{1-t \kappa_{j}}\right) v(t)=-1+\varphi^{t}\left(\kappa_{1}, \ldots, \kappa_{n-1}\right) v(t) .
$$

From estimate (46) we deduce that $v$ satisfies the differential inequality

$$
\left\{\begin{array}{l}
v^{\prime}(t) \geq-1+\frac{(n-1) K}{1-t K} v(t), \quad t \in[0, r] \\
v(r)=0
\end{array}\right.
$$

It is easily seen that the function $v_{0}:[0, r] \rightarrow \mathbb{R}$, defined in (45), is the solution to the Cauchy problem

$$
\begin{aligned}
& v_{0}^{\prime}(t)=-1+\frac{(n-1) K}{1-t K} v_{0}(t), \quad t \in[0, r], \\
& v_{0}(r)=0 .
\end{aligned}
$$

Since $v$ satisfies the differential inequality (47), we have

$$
0 \leq v(t) \leq v_{0}(t) \quad \forall t \in[0, r]
$$

Moreover,

$$
v_{0}^{\prime}(t)=-\frac{1}{n}\left[(n-1) \frac{(1-r K)^{n}}{(1-t K)^{n}}+1\right]<0 \quad \forall t \in[0, r],
$$

hence we conclude that

$$
0 \leq v(t) \leq \max _{[0, r]} v_{0}=v_{0}(0)=c(K, r),
$$

and the lemma is proven.

Proposition 5.9 Let $\Omega$ be a smooth $K$-admissible domain, and let $f \geq 0$ be a nonnegative bounded continuous function in $\Omega$. Then the function $v_{f}$ defined in (10) satisfies the bound

$$
0 \leq v_{f}(x) \leq\|f\|_{\infty, \Omega} c_{K}(d(x), \tau(x)), \quad \forall x \in \Omega,
$$

where

$$
c_{K}(d, \tau):= \begin{cases}\frac{(1-d K)^{n}-(1-(\mathrm{d}+\tau) K)^{n}}{n K(1-d K)^{n-1}}, & \text { if } K \neq 0 \\ \tau, & \text { if } K=0 .\end{cases}
$$

In particular, (42) holds. 
Proof From the explicit representation (10) of $v_{f}$, we have that

$$
0 \leq v_{f}(x) \leq\|f\|_{\infty, \Omega} \int_{d(x)}^{d(x)+\tau(x)} \prod_{i=1}^{n-1} \frac{1-s \kappa_{i}(x)}{1-d(x) \kappa_{i}(x)} \mathrm{d} s .
$$

The conclusion now follows from Lemma 5.8.

As a second step toward the proof of Theorem 5.3, we prove the analogous of Proposition 5.7 without the regularity assumptions on $\partial \Omega$ and $f$.

Proposition 5.10 The conclusions of Proposition 5.7 still hold under the assumptions of $\Omega \subset \mathbb{R}^{n}$ be a $K$-admissible domain, and $f \in L^{\infty}(\Omega)$ be a nonnegative function.

Proof For $j \in \mathbb{N}$, let $\Omega_{j}$ be a smooth $K_{j}$-admissible domain, such that the sequence $\left(\Omega_{j}\right)$ converges to $\Omega$ in the Hausdorff topology, and $\lim _{j} K_{j}=K$. Let us extend the functions $u_{\Omega_{j}}(x):=R d_{\Omega_{j}}(x)$ and $u_{\Omega}(x):=R d(x)$ to zero outside $\Omega_{j}$ and $\Omega$ respectively. (Here $d_{\Omega_{j}}$ denotes the distance function from the boundary of $\Omega_{j}$.) We extend in the same way the function $f$ outside $\Omega$. Let $B$ be a ball containing $\Omega$ and all the $\Omega_{j}$, and let $\left(f_{j}\right)_{j}$ be a sequence of Lipschitz continuous functions on $B$ satisfying

$$
\lim _{j}\left\|f_{j}-f\right\|_{L^{1}(B)}=0, \quad 0 \leq f_{j}(x) \leq\|f\|_{\infty} \quad \forall x \in B .
$$

Let us define

$$
\epsilon_{j}=\frac{c\left(K_{j}, r_{\Omega_{j}}\right)}{c\left(K, r_{\Omega}\right)}-1, \quad j \in \mathbb{N},
$$

where $c(K, r)$ is the quantity defined in (39). Since $\left(\Omega_{j}\right)$ converges to $\Omega$ in the Hausdorff topology, we have that $r_{\Omega_{j}} \rightarrow r_{\Omega}$ for $j \rightarrow+\infty$, hence $\lim _{j} \epsilon_{j}=0$. Let us define the functions

$$
h_{j}(s)=\left(1+\epsilon_{j}\right) h(s), \quad s \geq 0 .
$$

From the assumption (34) on $h$ we deduce that, for every $j$,

$$
h_{j}(R)=0, \quad h_{j}(s) \geq \max \left\{0,\left(1+\epsilon_{j}\right) \Lambda(s-R)\right\} .
$$

From the definition (50) of $\epsilon_{j}$, and the fact that $\left\|f_{j}\right\|_{\infty} \leq\|f\|_{\infty}$, it is straightforward to verify that

$$
\left\|f_{j}\right\|_{\infty} c\left(K_{j}, r_{\Omega_{j}}\right) \leq\|f\|_{\infty}\left(1+\epsilon_{j}\right) c\left(K, r_{\Omega}\right) \leq\left(1+\epsilon_{j}\right) \Lambda .
$$

From Proposition 5.7 we conclude that $u_{\Omega_{j}}=R d_{\Omega_{j}}$ is a minimizer of the functional

$$
J_{j}(u)=\int_{\Omega_{j}}\left[h_{j}(|D u(x)|)-f_{j}(x) u(x)\right] \mathrm{d} x
$$


in $W_{0}^{1,1}\left(\Omega_{j}\right)$. We recall that the sequence $\left(u_{\Omega_{j}}\right)$ converges uniformly to $u_{\Omega}$ in $B$. Since $\left(f_{j}\right)$ converges to $f$ in $L^{1}(B)$ we have that

$$
\begin{aligned}
\lim _{j} J_{j}\left(u_{\Omega_{j}}\right) & =-\lim _{j} \int_{\Omega_{j}} f_{j}(x) u_{\Omega_{j}}(x) \mathrm{d} x=-\lim _{j} \int_{B} f_{j}(x) u_{\Omega_{j}}(x) \mathrm{d} x \\
& =-\int_{B} f(x) u_{\Omega}(x) \mathrm{d} x=J_{0}\left(u_{\Omega}\right) .
\end{aligned}
$$

On the other hand, if $u \in W_{0}^{1,1}(\Omega)$ and if we set $u=0$ outside $\Omega$, we obtain

$$
\begin{aligned}
J_{0}(u) & =\int_{\Omega}[h(|D u|)-f(x) u] \mathrm{d} x=\lim _{j} \int_{\Omega_{j}}\left[h_{j}(|D u|)-f_{j}(x) u\right] \mathrm{d} x \\
& =\lim _{j} J_{j}(u) \geq \lim _{j} J_{j}\left(u_{\Omega_{j}}\right)=J_{0}\left(u_{\Omega}\right) .
\end{aligned}
$$

Hence $u_{\Omega}$ is a minimizer of $J_{0}$ in $W_{0}^{1,1}(\Omega)$, and the proof is complete.

We are now in a position to conclude the proof of Theorem 5.3.

Proof of Theorem 5.3 Let us fix $u \in W_{0}^{1,1}(\Omega)$, and prove that $J(u) \geq J\left(u_{\Omega}\right)$. Let us define the function

$$
f(x):= \begin{cases}\frac{g(x, u(x))-g\left(x, u_{\Omega}(x)\right)}{u(x)-u_{\Omega}(x)}, & \text { if } u(x) \neq u_{\Omega}(x), \\ 0, & \text { if } u(x)=u_{\Omega}(x) .\end{cases}
$$

From the very definition of $f$ and (38) we have that

$g(x, u(x))-g\left(x, u_{\Omega}(x)\right)=f(x)\left(u(x)-u_{\Omega}(x)\right), \quad 0 \leq f(x) \leq L, \quad$ a.e. $x \in \Omega$.

From Proposition 5.10 we have that $u_{\Omega}$ is a minimizer of the functional

$$
J_{0}(u)=\int_{\Omega}[h(|D u(x)|)-f(x) u(x)] \mathrm{d} x
$$

in $W_{0}^{1,1}(\Omega)$. Finally, we have that

$$
\begin{aligned}
J(u) & =J_{0}(u)+\int_{\Omega}\left[f(x) u_{\Omega}(x)-g\left(x, u_{\Omega}(x)\right)\right] \mathrm{d} x \\
& \geq J_{0}\left(u_{\Omega}\right)+\int_{\Omega}\left[f(x) u_{\Omega}(x)-g\left(x, u_{\Omega}(x)\right)\right] \mathrm{d} x=J\left(u_{\Omega}\right),
\end{aligned}
$$

and the proof is complete. 


\section{References}

1. Ambrosio, L.: Lecture notes on optimal transport problems. Mathematical aspects of evolving interfaces (Funchal, 2000), Lecture Notes in Math. 1812, pp. 1-52. Springer, Berlin, (2003)

2. Bardi, M., Capuzzo-Dolcetta, I.: Optimal control and viscosity solutions of HamiltonJacobi-Bellman equations. Systems and Control: Foundations and Applications. Boston, Birkhäuser (1997)

3. Boutreux, T., de Gennes, P.-G.: Surface flows of granular mixtures. I. General principles and minimal model. J. Phys. I France 6, 1295-1304 (1996)

4. Cannarsa, P., Cardaliaguet, P.: Representation of equilibrium solutions to the table problem for growing sandpile. J. Eur. Math. Soc. 6, 1-30 (2004)

5. Cannarsa, P., Sinestrari, C.: Semiconcave Functions, Hamilton-Jacobi equations, and optimal control. Progress in Nonlinear Differential Equations and Theyr Applications. Boston, Birkhäuser (2004)

6. Celada, P., Cellina, A.: Existence and non existence of solutions to a variational problem on a square. Houston J. Math. 24, 345-375 (1998)

7. Celada, P., Perrotta, S., Treu, G.: Existence of solutions for a class of non convex minimum problems. Math. Z. 228, 177-199 (1997)

8. Cellina, A.: Minimizing a functional depending on $\nabla u$ and on $u$. Ann. Inst. H. Poincaré, Anal. Non Linéaire 14, 339-352 (1997)

9. Clarke, F.H., Ledyaev, Yu. S., Stern, R.J., Wolenski, P.R.: Nonsmooth Analysis and Control Theory. New York, Springer (1998)

10. Crasta, G., Malusa, A.: Geometric constraints on the domain for a class of minimum problems. ESAIM Control Optim. Calc. Var. 9, 125-133 (2003)

11. Evans, L.C., Feldman, M., Gariepy, R.: Fast/slow diffusion and collapsing sandpiles. J. Differential Equations 137(1), 166-209 (1997)

12. Evans, L.C., Gangbo, W.: Differential equations methods for the Monge-Kantorovich mass transfer problem. Mem. Am. Math. Soc. 137(653), (1999)

13. Evans, L.C., Gariepy, R.F.: Measure Theory and Fine Properties of Functions. Studies in Advanced Mathematics. CRC Press, Boca Raton, FL (1992)

14. Federer, H.: Curvature measures. Trans. Am. Math. Soc. 93, 418-491 (1959)

15. Feldman, M.: Variational evolution problems and nonlocal geometric motion. Arch. Rational Mech. Anal. 146, 221-274 (1999)

16. Gilbarg, D., Trudinger, N.S.: Elliptic partial differential equations of second order. Grundlehren der Mathematischen Wissenschaften, 224. Berlin, Springer-Verlag (1983).

17. Hadeler, K.P., Kuttler, C.: Dynamical Models for Granular Matter. Granular Matter 2, 9-18 (1999)

18. Itoh, J., Tanaka, M.: The Lipschitz continuity of the distance function to the cut locus. Trans. Am. Math. Soc. 353(1), 21-40 (2001)

19. Li, Y.Y., Nirenberg, L.: The distance function to the boundary, Finsler geometry and the singular set of viscosity solutions of some Hamilton-Jacobi equations, Commun. Pure Appl. Math. 58, 85-146 (2005)

20. Lions, P.L.: Generalized Solutions of Hamilton-Jacobi Equations. Research Notes in Mathematics, vol. 69. Boston London Melbourne, Pitman (1982)

21. Prigozhin, L.: Variational model of sandpile growth. European J. Appl. Math. 7(3), 225-235 (1996)

22. Schneider, R.: Convex Bodies: The Brunn-Minkowski theory. Cambridge University Press, Cambridge (1993)

23. Treu, G.: An existence result for a class of non convex problems of the Calculus of Variations. J. Convex Anal. 5, 31-44 (1998)

24. Vornicescu, M.: A variational problem on subsets of $\mathbb{R}^{n}$. Proc. Roy. Soc. Edinburgh Sect. A 127, 1089-1101 (1997) 